

\section{DISCLAIMER}

This report was prepared as an account of work sponsored by an agency of the United States Government. Neither the United States Government nor any agency Thereof, nor any of their employees, makes any warranty, express or implied, or assumes any legal liability or responsibility for the accuracy, completeness, or usefulness of any information, apparatus, product, or process disclosed, or represents that its use would not infringe privately owned rights. Reference herein to any specific commercial product, process, or service by trade name, trademark, manufacturer, or otherwise does not necessarily constitute or imply its endorsement, recommendation, or favoring by the United States Government or any agency thereof. The views and opinions of authors expressed herein do not necessarily state or reflect those of the United States Government or any agency thereof. 


\section{DISCLAIMER}

Portions of this document may be illegible in electronic image products. Images are produced from the best available original document. 


\section{LEGAL NOTICE}

This report was prepared as an account of Government sponsored work. Neither the United Stetes, nor the Comimission, nor any person acting on behalf of the Commission:

A. Makes any warranty or representation, express or implied, with respect to the accuracy, completeness, or usefulness of the information contained in this report, or that the use of any information, apparatus, method, or process disclosed in this report may not infringe privately owned rights; or

B. Assumes any liabilities with respect to the use of, or for damages resulting from the use of information, apparatus, method, or process disclosed in this report.

As used in the above, "person acting on behalf of the Commission" includes any employee or contractor of the Commission, or employee of such contractor, to the extent that such employee or contractor of the Commission, or employee of such contractor prepares, disseminates, or provides access to, any information pursuant to his employment or contract with the Commission, or his employment with such contractor.

Printed in the United States of America

Available from

Clearinghouse for Federal Scientific and Technical Information

National Bureau of Standards, U.S. Department of Commerce

Springfield, Virginia 22151

Price: Printed Copy $\$ 3.00$; Microfiche $\$ 0.65$ 
This report was prepared as an account of Government sponsored work. Nelther the United
States, nor the Commission, nor any person acting on A. Makes any warranty or representation acting on behnlf of the Commission:

racy, completeness, or usefulness of the informpressed or implied, with respect to the accuof any information, apparatus, method, or proces contained in this report, or that the use or procesa disclosed in this report may not infiringe B. Assumes any liabilin

use of any information, apparatus, mespect to the use of, or for damages resulting from the As used in the above. "person acting or process disciosed in this report.

ployee or controction of the Commission" includes any such employee or or of the Commission, or such contractor to the exul emdisseminge commission, or employee of such contractor prept that

with the Commisslon, or his employment with such contractor.
.

\title{
CALCULATION OF REACTION RATES
}

IN

SMALL PHYSICAL REGIONS

BY MEANS OF

A NEW NON-MULTIGROUP ADJOINT

MONTE CARLO TECHNIQUE

\section{By:}

L. B. LEVITT

R. C. LEWIS

J. SPANIER*

*North American Rọckwell Science Center, Thousand Oaks, California

\section{ATOMICS INTERNATIONAL}

\author{
A DIVISION OF NORTH AMERICAN ROCKWELL. CORPORATION
}

CONTRACT: AT(04-3)-701

ISSUED: MARCH 15, 1969 


\section{DISTRIBUTION}

This report has been distributed according to the category

"Physics" as given in the Standard Distribution for Unclassified Scientific and Technical Reports, TID-4500. 


\section{CONTENTS}

Page

Abstract ............................ 4

I. Introduction. ...................... 5

II. Description of the Method .................... 7

III. Numerical Results . . . . . . . . . . . . . . . . . . 23

IV. Summary and Conclusions ................... 27

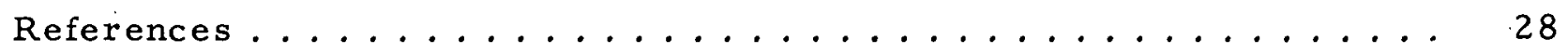

Appendices

I. Sampling From the Collision Density Equation. . . . . . . . 29

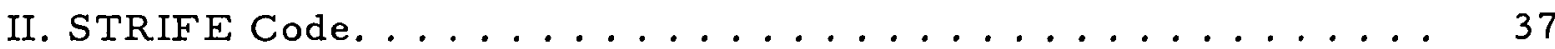

\section{TABLES}

1. Constant Cross-Section Results .................. 22

2. Energy-Dependent Cross-Section Results . ............ 25

\section{FIGURES}

1. Comparison of Relative Errors, Constant-Cross-Section Case.... 22

2. Computational Advantage of Adjoint Transformation . . . . . . . . 24

3. Comparison of Relative Errors, Two-Region Gold

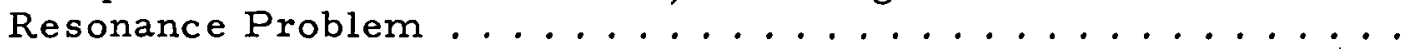




\begin{abstract}
The use of adjoint Monte Carlo in the study of reaction rates in small spatial regions of a reactor has an advantage over direct Monte Carlo techniques, in that it samples random walks from the "detector" (the small region under study) to the neutron source, rather than the reverse procedure which actually occurs. A new adjoint Monte Carlo technique is described which appears to be very useful for solving problems in the slowing-down region below the threshhold for inelastic scattering. The basis for the technique is the idea of exactly reversing direct Monte Carlo random walks, first used in the combined adjoint-direct Monte Carlo procedure developed for analysis of resonance integral experiments and discussed in NAA-SR-12494. The extension of the backward random walk to a complete adjoint Monte Carlo calculation permits the calculation of absolute reaction rates, whereas the combined technique was limited to relative reaction rates. The mathematical justification for this new technique must show that the resulting procedures exhibit some form of importance weighting. This is done by relating this technique to a multigroup adjoint technique (introduced by Gelbard and Spanier to solve problems in the thermal energy range) in such a way that it may be viewed as a logical extension of their work to continuous-energy slowingdown regions, thereby demonstrating a connection with dual importance sampling. Numerical results are provided which illustrate the gains in efficiency resulting from the use of this new technique.
\end{abstract}




\section{INTRODUCTION}

A frequent problem in nuclear reactor design is the estimation of the probability of an event of low frequency. For example, in the study of reaction rates in small spatial regions, interest focuses on the occasional particle which succeeds in entering the region. The practical use of Monte Carlo techniques to solve such problems often depends on the application of one form or another of importance sampling. (1-5) However, there is sometimes an alternative to the use of importance sampling as a means of improving the statistical uncertainty in the estimate of the answer. This alternative is the transforming of the original problem into one in which the event of interest occurs with relatively higher frequency than in the original problem. One may still, of course, use sophisticated sampling techniques in solving the auxiliary problem, thereby achieving even greater gains in efficiency.

One way in which such an auxiliary problem arises is through the solution of an adjoint transport equation. ${ }^{(6-11)}$ The reciprocity relationship shows how "source"-to-"detector" histories in the original problem may be replaced by "detector"-to-"source" histories of an adjoint simulation in the estimation of key design parameters. Then, in cases where the detector region is small (perhaps even a point), the adjoint simulation might be expected to yield more efficient estimates, provided that the source region is "larger" than the detector region. The word "larger" is used here in the sense of probability. The present report describes a new adjoint technique which appears to be very useful for solving problems in the slowing-down region.

The utility of the backward random walk developed in the TRAFE code $(12)$ was found to extend to complete adjoint solutions of similar problems. While the former technique was very useful in resonance integral experiments, it could not be applied to resonance escape probability calculations; nor could one see, from that point of view, the extension to anisotropic elastic scattering. The mathematical justification for using the backward random walk in an adjoint formulation must show that the resulting procedures exhibit some form of importance weighting. We have been able to show this in a.manner which will be made more precise later by relating this technique to a multigroup adjoint technique 
used by Gelbard and Spanier $(8,9,11)$ to solve problems in the thermal energy range. By viewing this technique as a logical extension of the work of Gelbard and Spanier to continuous energy slowing-down regions, we have been able to demonstrate a connection with dual importance sampling. While we have used the calculation of the resonance escape probability in foils to demonstrate this new technique, it is apparent that it is equally useful for calculating reaction rates in any localized region. The speed of the calculation is such that parametric studies can be performed efficiently, enabling this method to be used not only in analyzing experiments but in verifying or improving analytic approximations to quantities such as self-shielding factors and resonance integrals. In addition, the adjoint technique described in this report has the advantage over other suggested methods in that it may be based on the same cross-section library as would be used to solve problems by direct Monte Carlo methods.

Since it is our hope that this method will be adapted for use in practical problems and extended to more complex physical problems, we are presenting, in this report, a mathematical and physical basis for this technique from several points of view. In the main body of this report, we relate an adjoint integrodifferential equation utilizing a discontinuous flux transformation to an integral equation adjoint to the integral transport equation for the density of particles emerging from collision. In this way, we were able to connect this work to the previously mentioned work of Gelbard and Spanier. In Appendix I, we show how the same technique may be thought of as sampling from an integral equation adjoint to the integral transport equation for the particle collision density, utilizing a biased scattering kernel which is normalized for the "upscattering" of particles in the adjoint mode. It was, in fact, the manner in which the methods developed and applied in the TRAFE code were extended to complete adjoint methods. We feel that the understanding of the method will be enhanced by this dual presentation, thereby encouraging more widespread use.

A detailed description of the STRIFE code is included in Appendix II because, while conceived for calculation of our idealized test problems, it can in fact be easily adapted in its present form or with minor modifications, to the solution of practical problems involving reaction rates in localized regions. 


\section{DESCRIPTION OF THE METHOD}

In this section, we give both an intuitive and a more formal description of the new technique. We begin with the integral equation for the density, $X(\underline{x}, E, \underline{\omega})$, of particles emerging from a collision (or the source),

$$
X(\underline{x}, E, \underline{\omega})=\iiint x\left(\underline{x}^{\prime}, E^{\prime}, \underline{\omega}^{\prime}\right) T\left(\underline{x}, \underline{x} ; E^{\prime}, \underline{\omega}^{\prime}\right) C\left(E^{\prime}, \underline{\omega^{\prime}}, E, \underline{\omega} ; \underline{x}\right) d x^{\prime} d E^{\prime} d \omega^{\prime}+Q(\underline{x}, E, \underline{\omega})
$$

In Equation 1, $Q(\underline{x}, E, \underline{\omega})$ is the slowing-down source to the problem; $T\left(\underline{x^{\prime}}, \underline{x} ; E^{\prime}, \underline{\omega}^{\prime}\right)$ is the transport kernel,

$$
\begin{gathered}
T\left(\underline{x^{\prime}}, \underline{x} ; E^{\prime}, \underline{\omega}^{\prime}\right)=\Sigma_{t}\left(\underline{x}, E^{\prime}\right) \exp \left[-\int_{0}^{\underline{\omega}^{\prime} \cdot\left(\underline{x}-\underline{x}^{\prime}\right)} \Sigma_{t}\left(\underline{x}^{\prime}+s \underline{\omega}^{\prime}, E^{\prime}\right) d s\right] \delta\left[\underline{\omega}_{1} \cdot\left(\underline{x}-\underline{x}^{\prime}\right)\right] \\
\cdot \delta\left[\underline{\omega}_{2} \cdot\left(\underline{x}-\underline{x}^{\prime}\right)\right] \eta\left[\underline{\omega}^{\prime} \cdot\left(\underline{x}-\underline{x}^{\prime}\right)\right],
\end{gathered}
$$

where $\Sigma_{t}$ is the total macroscopic cross section, $\underline{\omega}^{\prime}, \underline{\omega}_{1}$, and $\underline{\omega}_{2}$ form an orthonormal triple of vectors, and $\eta(t)=1$ if $t>0, \eta(t)=0$ if $t \leq 0$; and $C\left(E^{\prime}, \underline{\omega}, E, \underline{\omega} ; \underline{x}\right)$ is the collision kernel,

$$
C\left(E^{\prime}, \underline{\omega^{\prime}}, E, \underline{\omega} ; \underline{x}\right)=\frac{\Sigma_{s}\left(\underline{x}, E^{\prime}\right)}{\Sigma_{t}\left(\underline{x}, E^{\prime}\right)} P\left(E^{\prime}, \underline{\omega^{\prime}}, E, \underline{\omega} ; \underline{x}\right),
$$

where $P$ is a properly normalized probability density function for scattering into energy $E$ and direction $\underline{\omega}$ at $\underline{x}$. For scattering which is elastic, and isotropic in the center of mass (CM) system,

$$
\begin{aligned}
P\left(E^{\prime}, \underline{\omega}, E, \underline{\omega} ; \underline{x}\right) & =\frac{1}{E^{\prime}(1-\alpha)} \delta\left(\mu_{L}-\frac{A \mu_{c}+1}{\sqrt{A^{2}+2 A \mu_{c}+1}}\right) \\
& \text { for } \alpha E^{\prime} \leq E \leq E^{\prime}, \\
& =0 \text { otherwise, }
\end{aligned}
$$

*See, for example, Reference 13, Appendix 2 
where $\mu_{L}=\underline{\omega} \cdot \underline{\omega}^{\prime}$ is the cosine of the scattering angle in the laboratory system, $\mu_{\mathrm{c}}=\left[2\left(\mathrm{E} / \mathrm{E}^{\prime}\right)-(1+\alpha)\right] /(1-\alpha)$ is the cosine of the scattering angle in the CM.system, $\alpha=[(\mathrm{A}-1) /(\mathrm{A}+1)]^{2}$, and $\mathrm{A}$ is the mass of the scattering nucleus at $\mathrm{x}$. In Equation 4 and all subsequent references to $P$ a factor $1 / 2 \pi$, corresponding to a uniform choice of azimuthal angle, has been omitted for convenience. None of our conclusions is affected by this omission.

If one wishes to calculate, for example, an absorption rate over a volume, $R$, of phase space, this absorption rate may be written

$$
\begin{aligned}
I & =\iiint_{R} \frac{\sum_{a}(\underline{x}, E)}{\sum_{t}(\underline{x}, E)} \psi(\underline{x}, E, \underline{\omega}) d \underline{x} d E d \underline{\omega} \\
& =\iiint\left[\int_{R} \frac{\Sigma_{a}(\underline{x}, E)}{\Sigma_{t}(\underline{x}, E)} X\left(\underline{x}^{\prime}, E, \underline{\omega}\right) T\left(\underline{x^{\prime}}, x ; E, \underline{\omega}\right) d \underline{x}^{\prime}\right] d \underline{x} d E d \underline{\omega},
\end{aligned}
$$

where $\Sigma_{a}$ is the macroscopic absorption cross section and $\psi(\underline{x}, E, \underline{\omega})=\Sigma_{t}(\underline{x}, E) F(\underline{x}, E, \underline{\omega})$ is the vector collision density, $F$ the vector flux. Then the equation adjoint to the pair of equations ( 1 and 5 ) is

$$
\begin{aligned}
& X^{*}(\underline{x}, E, \underline{\omega})=\iiint X^{*}\left(\underline{x}^{\prime}, E^{\prime}, \underline{\omega^{\prime}}\right) \mathrm{T}\left(\underline{\mathbf{x}}, \underline{x}^{\prime} ; E, \underline{\omega}\right) C\left(E, \underline{\omega}, E^{\prime}, \underline{\omega}^{\prime} ; \underline{x}^{\prime}\right) \mathrm{d} \underline{x}^{\prime} \mathrm{d} E^{\prime} \mathrm{d} \underline{\omega}^{\prime} \\
& +\int_{R}^{\sum_{\mathrm{a}}\left(\underline{x}^{\prime}, \mathrm{E}\right)} \frac{\mathrm{t}}{\sum_{\mathrm{t}}\left(\underline{x}^{\prime}, \mathrm{E}\right)} \mathrm{T}\left(\underline{\mathrm{x}}, \underline{x}^{\prime} ; \mathrm{E}, \underline{\omega}\right) \mathrm{d} \underline{x}^{\prime},
\end{aligned}
$$

and the integral which corresponds to Equation 5 is

$$
I *=\iiint Q(\underline{x}, E, \underline{\omega}) X^{*}(\underline{x}, E, \underline{\omega}) d \underline{x} d E d \underline{\omega} .
$$

Upon multiplying Equation 1 by $X *(\underline{x}, E, \underline{\omega})$ and Equation 6 by $X(\underline{x}, E, \underline{\omega})$ and integrating these equations, one sees that

$$
I=I^{*} \text {. }
$$


This suggests that the absorption rate, I, may be estimated in two different ways. One might construct Monte Carlo estimates of the density, $X$; through a simulation of Equation 1 and use any estimator whose mean is the fraction of particles absorbed in $\mathrm{R}$. Alternatively, one might construct Monte Carlo estimates of the density, $X^{*}$, through a simulation of Equation 6 and use any estimator whose mean is the weighted fraction which arrives at the source region defined by $Q \neq 0$. Such simulations are, of course, subject to an interpretation of Equations 1 and 6 as proper transport equations, a point which we now discuss more fully.

Estimation of I through a simulation of Equation 1 is, of course, straightforward. Because the functions $Q, T$, and $P$ are properly normalized density functions, one may select a starting position $\underline{x}_{0}$, energy $E_{0}$, and direction $\underline{\omega}_{0}$. from the density $Q$, move the particle along $\underline{\omega}_{0}$ to its first collision at point $\underline{x}_{1}$ chosen from the density $\mathrm{T}\left(\underline{x}_{0}, \underline{x}_{1} ; E_{0}, \underline{\omega}_{0}\right)$, absorb the particle at $\underline{x}_{1}$ with probability $1-\Sigma_{s}\left(\underline{x}_{1}, E_{0}\right) / \Sigma_{t}\left(\underline{x}_{1}, E_{0}\right)$ or scatter it with probability $\Sigma_{s}\left(\underline{x}_{1}, E_{0}\right) /$ $\Sigma_{t}\left(\underline{x}_{1}, E_{0}\right)$, to a new energy $E_{1}$ and direction $\underline{\omega}_{1}$ selected from the density $P$. The simulation continues through alternate selections from $T$ and $P$ until termination occurs in some region. In the particle population so constructed, the density emerging from collisions is an estimate of $X(\underline{x}, E, \underline{\omega})$, and a valid estimator of $I$ is the fraction of particles absorbed in $E$ or equivalently, the sum over all collisions of the expected contributions to the absorption rate in $R$ for a particle which has just emerged from collision.

A proper simulation of Equation 6, however, is more complicated, because of the fact that the adjoint source, transport kernel, and collision kernel are not properly normalized. In fact, it is somewhat nontrivial even to sample the adjoint source function

$$
Q *(\underline{x}, E, \underline{\omega})=\int_{R}^{\Sigma_{a}\left(\underline{x^{\prime}}, E\right)} \frac{\Sigma_{t}\left(\underline{x^{\prime}}, E\right)}{\sum^{\prime}} T\left(\underline{x}, \underline{x}^{\prime} ; E, \underline{\omega}\right) d \underline{x}^{\prime} .
$$

Physically, the spatial positions $\underline{x}$ in $Q *$ reppresent scattering points from which particles travel along $\underline{\omega}$ to an absorbing event in $R$. One might sample for such an $x$ through a rejection technique; but this would be very inefficient if $R$ were small. A more attractive alternative is to start the particle uniformly in $R$ and 
trace it back to the point of its previous collision. Thus, if $\underline{\omega}_{0}$ is selected from an isotropic distribution, $E_{0}$ from

$$
\frac{\Sigma_{a}\left(\underline{x}_{o}, E\right)}{\Sigma_{t}\left(\underline{x}_{o}, E\right)} / \int \mathrm{dE} \frac{\Sigma_{a}\left(\underline{x}_{o}, E\right)}{\Sigma_{t}\left(\underline{x}_{o}, E\right)}, \underline{x}_{o} \in R,
$$

where $\underline{x}_{0}$ is selected uniformly in $R$, then $\underline{x}_{1}$ may be chosen from the density

$$
T\left(\underline{x}_{0}, \underline{x}_{1} ; E_{0}, \underline{\omega}_{0}\right)=T\left(\underline{x}_{1}, \underline{x}_{0} ; E_{0},-\underline{\omega}_{0}\right) \frac{\Sigma_{t}\left(\underline{x}_{1}, E_{o}\right)}{\sum_{t}\left(\underline{x}_{0}, E_{0}\right)} .
$$

Thus, a particle with coordinates $\left(\underline{x}_{1}, E_{0}, \underline{\omega}_{0}\right)$ selected in this manner, will represent a sample drawn from $Q *(\underline{x}, E,-\underline{\omega})$, provided it is assigned a weight

$$
\mathrm{W}_{\mathrm{o}}=4 \pi \mathrm{V}_{\mathrm{R}}\left[\int \frac{\Sigma_{\mathrm{a}}\left(\underline{x}_{0}, E\right)}{\Sigma_{\mathrm{t}}\left(\underline{x}_{0}, E\right)} \mathrm{dE}\right] \frac{\Sigma_{\mathrm{t}}\left(\underline{x}_{0}, E_{o}\right)}{\Sigma_{\mathrm{t}}\left(\underline{x}_{1}, E_{0}\right)},
$$

where $V_{R}=$ volume of $R$.

The reversal of direction in $Q *$ then occurs in a very natural way, and suggests that we might more easily simulate the density $\chi^{*}(\underline{x}, E,-\underline{\omega})$ [replacing $\underline{\omega}$ by $-\underline{\omega}$ in Equation 6] than the density $x^{*}(\underline{x}, E, \underline{\omega})$. We will still, of course, obtain $I *$ by replacing $\underline{\omega}$ by $\underline{-\omega}$ in Equation 7 as well. The equation satisfied by $X^{*}(\underline{x}, \mathrm{E},-\underline{\omega})$ is

$$
\begin{aligned}
& X^{*}(\underline{x}, E,-\underline{\omega})=\iiint X^{* *}\left(\underline{x}^{\prime} E^{\prime},-\underline{\omega}^{\prime}\right) \mathrm{T}\left(\underline{x}, \underline{x}^{\prime} ; E,-\underline{\omega}\right) C\left(E, \underline{\omega}, E^{\prime}, \underline{\omega}^{\prime}, \underline{x}^{\prime}\right) \mathrm{d} \underline{x}^{\prime} \mathrm{d} E^{\prime} \mathrm{d} \underline{\omega}^{\prime} \\
& +\int_{R} \frac{\sum_{a}\left(\underline{x}^{\prime}, E\right)}{\sum_{t}\left(\underline{x}^{\prime}, E\right)} T\left(\underline{x}, \underline{x}^{\prime} ; E,-\underline{\omega}\right) d \underline{x^{\prime}} .
\end{aligned}
$$

Notice that we have made use of the fact that the collision kernel, C, depends only on $\underline{\omega} \cdot \underline{\omega}^{\prime}=(-\underline{\omega}) \cdot\left(-\underline{\omega}^{\prime}\right)$. 
Pursuing this line of thought, upon collision at $\underline{x}_{1}$ we may choose to absorb the particle with probability $\Sigma_{\mathrm{a}}\left(\underline{x}_{1}, E_{0}\right) / \Sigma_{t}\left(\underline{x}_{1}, E_{0}\right)$ and, with probability $\Sigma_{s}\left(\underline{x}_{1}, E_{0}\right) / \Sigma_{t}\left(\underline{x}_{1}, E_{0}\right)$, to scatter into a new direction $\underline{\omega}_{1}$ and energy $E_{1}$. To perform the transformation in energy and direction, we must sample $E_{1}, \underline{\omega}_{1}$ from

$$
\begin{aligned}
\frac{1}{W_{c}} C\left(E_{1}, \underline{\omega}_{1}, E_{o}, \underline{\omega}_{o} ; \underline{x}_{1}\right) & =\frac{1}{W_{c}} \frac{\sum_{s}\left(\underline{x}_{1}, E_{1}\right)}{\sum_{t}\left(\underline{x}_{1}, E_{1}\right)} \frac{1}{E_{1}(1-\alpha)} \delta\left(\mu_{L}-\frac{A \mu_{c}+1}{\sqrt{A^{2}+2 A \mu_{c}+1}}\right), \\
& \text { for } E_{o} \leq E_{1} \leq E_{o} / \alpha, \\
& =0 \text { otherwise, }
\end{aligned}
$$

and use a weight

$$
\begin{aligned}
\mathrm{w}_{c} & =\frac{\iint \mathrm{C}\left(\mathrm{E}_{1}, \underline{\underline{\omega}}_{1}, \mathrm{E}_{0}, \underline{\omega}_{0} ; \underline{x}_{1}\right) \mathrm{d} E_{1} \mathrm{~d} \underline{\underline{\omega}}_{1}}{\Sigma_{s}\left(\underline{x}_{1}, E_{0}\right) / \Sigma_{t}\left(\underline{x}_{1}, E_{0}\right)} \\
& =\int_{E_{0}}^{E_{0} / \alpha} \frac{\Sigma_{s}\left(\underline{x}_{1}, E_{1}\right) d E_{1}}{\Sigma_{t}\left(\underline{x}_{1}, E_{1}\right) E_{1}(1-\alpha)} / \Sigma_{s}\left(\underline{x}_{1}, E_{0}\right) / \Sigma_{t}\left(\underline{x}_{1}, E_{0}\right) .
\end{aligned}
$$

Having arrived at $\left(\underline{x}_{1}, E_{1}, \underline{\omega}_{1}\right)$ in this fashion, we next sample for $\underline{x}_{2}$ from

$$
\mathrm{T}\left(\underline{\mathbf{x}}_{1}, \underline{\mathbf{x}}_{2} ; \mathrm{E}_{1}, \underline{\omega}_{1}\right)=\mathrm{T}\left(\underline{\mathbf{x}}_{2}, \underline{\mathbf{x}}_{1} ; \mathrm{E}_{1},-\underline{\omega}_{1}\right) \frac{\Sigma_{\mathrm{t}}\left(\underline{\mathbf{x}}_{2}, \mathrm{E}_{1}\right)}{\Sigma_{t}\left(\underline{\mathbf{x}}_{1}, \mathrm{E}_{1}\right)},
$$

making use of the weight

$$
\mathrm{W}_{\mathrm{T}}=\frac{\Sigma_{\mathrm{t}}\left(\underline{x}_{1}, E_{1}\right)}{\Sigma_{\mathrm{t}}\left(\underline{x}_{2}, E_{1}\right)} .
$$


Sampling the adjoint kernels, $C$ and $T$, alternates until the particle is absorbed, escapes from the system, or is scattered above energies of interest. Then the product of the particle's weight and $Q(\underline{x}, E,-\underline{\omega})$ produces an unbiased estimate of $I *_{*}=I$. We emphasize that the density being constructed in this adjoint simulation is: $X^{*}(\underline{x}, E, \underline{w})$, rather than the solution of Equation 6 .

This same difficulty, that of constructing Monte Carlo solutions of equations with unnormalized kernels, becomes even more apparent in working with the integro-differential equation for the vector flux, $F(\underline{x}, E, \underline{\omega})$. This transport equa tion may be written

$\underline{\omega} \cdot \underline{\nabla} F(\underline{x}, E, \underline{\omega})+\Sigma_{t}(\underline{x}, E) F(\underline{x}, E, \underline{\omega})=\iint \Sigma_{t}\left(\underline{x}, E^{\prime}\right) C\left(E^{\prime}, \underline{\omega}^{\prime}, E, \underline{\omega} ; \underline{x}\right) F\left(\underline{x}, E^{\prime}, \underline{\omega}^{\prime}\right) d E^{\prime} d \underline{\omega}^{\prime}$

$$
\begin{aligned}
& +\mathrm{Q}(\underline{\mathrm{x}}, \mathrm{E}, \underline{\omega}) \\
& =\iint \Sigma_{\mathrm{s}}\left(\underline{\mathrm{x}}, \mathrm{E}^{\prime}\right) \mathrm{P}\left(\mathrm{E}^{\prime}, \underline{\omega^{\prime}}, \mathrm{E}, \underline{\omega} ; \underline{\mathrm{x}}\right) \mathrm{F}\left(\underline{\mathrm{x}}, \mathrm{E}^{\prime}, \underline{\omega}^{\prime}\right) \mathrm{d} \mathrm{E}^{\prime} \mathrm{d} \underline{\omega}^{\prime} \\
& \quad+\mathrm{Q}(\underline{\mathrm{x}}, \mathrm{E}, \underline{\omega}) .
\end{aligned}
$$

The adjoint integro-differential equation is (cf. Reference 11, p $184 \mathrm{ff}$ )

$-\underline{\omega} \cdot \underline{\nabla} F *(\underline{x}, E, \underline{\omega})+\Sigma_{t}(\underline{x}, E) F *(\underline{x}, E, \underline{\omega})=\iint \Sigma_{s}(\underline{x}, E) P\left(E, \underline{\omega}, E^{\prime}, \underline{\omega}^{\prime} ; \underline{x}\right) F *\left(\underline{x}, E^{\prime}, \underline{\omega}^{\prime}\right) d E^{\prime} d \underline{\omega}^{\prime}$

$$
+Q * * * x, E, \underline{\omega})
$$

Then, with suitable boundary conditions on $F$ and $F *$, we have

$$
\iint Q * *(\underline{x}, E, \underline{\omega}) F(\underline{x}, E, \underline{\omega}) d \underline{x} d E d \underline{\omega}=\iint Q(\underline{x}, E, \underline{\omega}) F *(\underline{x}, E, \underline{\omega}) d \underline{x} d E d \underline{\omega} .
$$

For the problem we are studying, Equation 5 implies that

$$
Q * *(\underline{x}, E, \underline{\omega})=\left\{\begin{array}{l}
\Sigma_{a}(\underline{x}, E), \underline{x} \in R \\
0, \text { otherwise. }
\end{array}\right.
$$


One difficulty with constructing a simulation of Equation 16 arises because of the $-\underline{\omega}$ in the leakage term. To circumvent this difficulty we use the same tactic as before; we define

$$
F^{\dagger}(\underline{x}, E ; \underline{\omega})=F^{*}(\underline{x}, E,-\underline{\omega})
$$

The replacement of $\underline{\omega}$ by $-\underline{\omega}$ in Equation 16 gives

$$
\begin{aligned}
\underline{\omega} \cdot \underline{\nabla} F^{\dagger}(\underline{x}, E, \underline{\omega})+\Sigma_{t}(\underline{x}, E) F^{\dagger}(\underline{x}, E, \underline{\omega}) & =\iint \Sigma_{s}(\underline{x}, E) P\left(E, \underline{\omega}, E^{\prime}, \underline{\omega}^{\prime} ; \underline{x}\right) F^{\dagger}\left(\underline{x}, E^{\prime}, \underline{\omega}^{\prime}\right) d E^{\prime} d \underline{\omega}^{\prime} \\
& +Q^{*} *(\underline{x}, E,-\underline{\omega}) .
\end{aligned}
$$

an equation which may be readily simulated, and which leads to a model different from, but equivalent to, the one already described. We would expect this equivalence, since the equality

$$
\iint \mathrm{QF} *=\iint \mathrm{Q} \mathrm{X}^{*}
$$

for all source functions, $Q$, implies that

$$
\dot{F} *=X^{*}
$$

Therefore, a simulation of the function $X^{*}(\underline{x}, E,-\underline{\omega})$ must ultimately be statistically equivalent to a simulation of $F^{*}(\underline{x}, E,-\underline{\omega})=F^{\dagger}(\underline{x}, E, \underline{\omega})$.

Based on the integro-differential Equation 18, which is now a conventional transport equation, a sampling procedure which suggests itself is the following: Select a starting direction, $\underline{\omega}_{0}$, from an isotropic distribution, $\underline{x}_{0}$ uniformly in $R$, and $E_{o}$ from $\Sigma_{a}\left(\underline{x}_{0}, E\right) / \int d E \Sigma_{a}\left(\underline{x}_{0}, E\right)$. The source weight is

$$
\mathrm{w}_{\mathrm{o}}^{\prime}=4 \pi \mathrm{V}_{\mathrm{R}} \int \Sigma_{\mathrm{a}}\left(\underline{x}_{0}, E\right) \mathrm{dE} .
$$

Next, the position, $\underline{x}_{1}$, of first collision is chosen from $T\left(\underline{x}_{0}, \underline{x}_{1} ; E_{0}, \underline{\omega}_{0}\right)$. At $\underline{x}_{1}$, absorption occurs with probability $\Sigma_{a}\left(\underline{x}_{1}, E_{0}\right) / \Sigma_{t}\left(\underline{x}_{1}, E\right)$, and scattering with probability $\Sigma_{s}\left(\underline{x}_{1}, E_{0}\right) / \Sigma_{t}\left(\underline{x}_{1}, E_{o}\right)$. 
In the latter event, a new energy, $E_{1}$, and direction, $\underline{\omega}_{1}$, are selected from

$$
\begin{aligned}
& \frac{1}{W_{c}^{\prime} \cdot \Sigma_{s}\left(\underline{x}_{1}, E_{0}\right)} \Sigma_{t}\left(\underline{x}_{1}, E_{1}\right) C\left(E_{1}, \underline{\omega}_{1}, E_{0}, \underline{\omega}_{0} ; \underline{x}_{1}\right) \\
& =\frac{1}{W_{c}^{\prime} \cdot \Sigma_{s}\left(\underline{x}_{1}, E_{0}\right)} \frac{\Sigma_{s}\left(\underline{x}_{1}, E_{1}\right)}{E_{1}(1-\alpha)} \delta\left(\mu_{L}-\frac{A \mu_{c}+1}{\sqrt{A^{2}+2 A \mu_{c}+1}}\right), \\
& \text { for } E_{0} \leq E_{1} \leq E_{0} / \alpha \\
& =0 \text { otherwise, }
\end{aligned}
$$

and a multiplicative weight factor

$$
\mathrm{w}_{c}^{\prime}=\int_{E_{0}}^{E_{0} / \alpha} \frac{\Sigma_{s}\left(\underline{x}_{l}, E_{1}\right) d E_{1}}{E_{l}(1-\alpha)} / \Sigma_{s}\left(\underline{x}_{1}, E_{0}\right)
$$

is assigned. This alternation between collision and transport kernels continues until the particle is absorbed, escapes from the system, or is scattered above energies of interest. This sampling procedure may be shown to be statistically equivalent $\S$ to the one based on the integral equation for $x^{*}$, described earlier.

$\S$ For example, if we denote the source density

$$
\frac{\Sigma_{a}\left(\underline{x}_{0}, E\right)}{\Sigma_{t}\left(\underline{x}_{0}, E\right)} \int \frac{\Sigma_{a}\left(\underline{x}_{0}, E\right)}{\sum_{t}\left(\underline{x}_{0}, E\right)} d E
$$

by $f_{0}$, and the source density

$$
\Sigma_{\mathrm{a}}\left(\underline{x}_{0}, E\right) / \int \Sigma_{\mathrm{a}}\left(\underline{x}_{0}, E\right) d E
$$

by $f_{o}^{\prime}$, then drawing a sample energy, $E_{o}$, from $f_{o}$ with weight

$$
\int \frac{\Sigma_{a}\left(\underline{x}_{0}, E\right)}{\Sigma_{t}\left(\underline{x}_{0}, E\right)} d E
$$

is equivalent to drawing a sample energy, $E_{o}^{\prime}$, from $f_{0}^{\prime}$ with weight

$$
\int \frac{\Sigma_{a}\left(\underline{x}_{o}, E\right)}{\Sigma_{t}\left(\underline{x}_{o}, E\right)} d E \cdot \frac{f_{o}\left(E_{o}^{\prime}\right)}{f_{o}^{\prime}\left(E_{o}^{\prime}\right)}=\frac{\Sigma_{a}\left(\underline{x}_{o}, E_{o}^{\prime}\right)}{\Sigma_{t}\left(\underline{x}_{o}, E_{o}^{\prime}\right)} \cdot \frac{\int \Sigma_{a}\left(\underline{x}_{o}, E\right) d E}{\Sigma_{a}\left(\underline{x}_{0}, E_{o}^{\prime}\right)}=\frac{\int \Sigma_{a}\left(\underline{x}_{o}, E\right) d E}{\Sigma_{t}\left(\underline{x}_{0}, E_{o}^{\prime}\right)} .
$$

Applying the same idea to the sampling from the two different collision kernels completes the proof of the equivalence of the two sampling procedures. 
While the two "analog" adjoint sampling and weighting procedures just described lead to the same expected values, they do, in general, have different variances. Furthermore, the weight factors, $\mathrm{W}_{\mathrm{c}}$ (Equation 12) $\mathrm{x}_{\mathrm{T}}$ (Equation 14) and $W_{c}^{\prime}$ (Equation 21), result in significantly larger variances than would arise in direct mode simulation. As is observed in Reference 11 , this appears to be true precisely because the weights seem in no way to reflect the importance of the event being weighted.

One may ask whether the use of appropriately altered collision and transport kernels in an adjoint mode simulation might eliminate some or all of this additional variance. We can get some feeling for the need for such improvement by examining a constant-cross-section model. Under this assumption, the two analog adjoint sampling schemes become identical, and the weight factor arising from collision at an energy $E_{l}$ (assuming isotropic scattering in the CM system) is

$$
W=\int_{E_{0}}^{E_{0} / \alpha} \frac{d E_{1}}{E_{1}(1-\alpha)}=\frac{\ln \frac{1}{\alpha}}{1-\alpha} .
$$

It seems clear that, even in this relatively simple case, the variability of the total weight with the number of collisions, as well as with the mass of the scatterers present, will cause a harmful effect on the variance. This difficulty may be virtually eliminated by a physically intuitive biasing (see Reference 13) of the adjoint scattering kernel.

To see this, we examine the energy-angle relationship for elastic scattering,

$$
E_{L}=\frac{E_{U}}{2}\left[(1+\alpha)+(1-\alpha) \mu_{c}\right] \text {, }
$$

where $E_{L}$ and $E_{U}$ designate lower and upper energies, respectively. In direct mode simulation, $\mu_{c}$ is determined through a random sampling of its density 
function, f, by setting a random number

$$
r=\int_{-1}^{\mu}{ }_{c} f(\mu) d \mu,
$$

after which, since $E_{U}$ is known, $E_{L}$ is determined through Equation 23. If one could envision a filmed sequence depicting this collision in time, it would seem natural to regard the adjoint collision as what would be seen if the film were run backward. This implies that the scattering angle in the CM system should be preserved (i.e. selected by means of Equation 24), as in a direct mode collision. This would suggest the use of a biased scattering kernel, equal to $E_{L} / E_{U}$ times the normalized probability of slowing down from $E_{U}$ to $E_{L}$. The factor $E_{L} / E_{U}$ properly normalizes the kernel for adjoint sampling of the upper energy, $E_{U}$, and may be applied to any elastic angular distribution $f(\mu)$, as long as $f(\mu)$ is independent of energy.

It remains to be seen that the use of this altered scattering kernel removes most of the extra variance caused by the factor $W_{c}^{\prime}$ (Equation 21 ). One also may wonder whether the use of still other kernels might not be equally good, or perhaps even better, from the standpoint of variance. Both of these questions may be settled by examining transformations of the adjoint transport equation designed to achieve unit weight factors, as we now do.

We begin with the integro-differential Equation 18 for the function $\mathrm{F}^{\dagger}$, which is related to the adjoint flux, $F *$, through Equation 17. We set ourselves the goal of finding a transformation of Equation 18 which will lead to an adjoint equation which can be simulated without the need for weights on every collision. We shall discover a transformation which avoids weights, except at boundary crossings between regions of different scattering cross section, and which is equivalent to a time reversal of the direct mode collision.

Let $\psi^{\infty}(\underline{x}, E, \underline{\omega})$ be a prospective transforming function, and multiply Equation 18 by $\psi^{\infty}$; further, let

$$
\eta(\underline{x}, E, \underline{\omega}) \equiv \mathrm{F}^{\dagger}(\underline{x}, \mathrm{E}, \underline{\omega}) \psi^{\infty}(\underline{\mathrm{x}}, \mathrm{E}, \underline{\omega})
$$


Then Equation 18 becomes

$\underline{\omega} \cdot \underline{\nabla} \eta(\underline{x}, E, \underline{\omega})+\Sigma_{t}(\underline{x}, E) \eta(\underline{x}, E, \underline{\omega})=\iint \frac{\psi^{\infty}(\underline{x}, E, \underline{\omega})}{\psi^{\infty}\left(\underline{x}, E^{\prime}, \underline{\omega}^{\prime}\right)} \Sigma_{s}(\underline{x}, E) P\left(E, \underline{\omega}, E^{\prime}, \underline{\omega}^{\prime} ; \underline{x}\right) \eta\left(\underline{x}, E^{\prime}, \underline{\omega}^{\prime}\right) d E^{\prime} d \underline{\omega}^{\prime}$

$$
+\psi^{\infty}(\underline{x}, E, \underline{\omega}) Q * *(\underline{x}, E,-\underline{\omega})
$$

If the integral which appears on the right hand side is to be interpreted as a scattering rate at $\left(\underline{x}, E^{\prime}, \underline{\omega}^{\prime}\right)$, and if unit weights are demanded on every collision (i.e., scattering-in rate = scattering-out rate), then we must have

$$
\Sigma_{s}\left(\underline{x}, E^{\prime}\right)=\iint_{\psi^{\infty}\left(\underline{x}, E, \underline{\omega}^{\prime}\right)}^{\left.E^{\prime}, \underline{\omega}^{\prime}\right)} \Sigma_{s}(\underline{x} ; E) P\left(E, \underline{\omega}, E^{\prime}, \underline{\omega}^{\prime} ; \underline{x}\right) \mathrm{dE} d \underline{\omega} .
$$

In a problem consisting of an infinite lattice of two regions, I and II, then we would want, for $\underline{x} \in I$,

$$
\Sigma_{s I}\left(\underline{x}, E^{\prime}\right)=\iint_{\psi^{\infty}(\underline{x}, E, \underline{\omega})}^{\psi^{\infty}\left(\underline{x}, E^{\prime}, \underline{\omega}^{\prime}\right)} \Sigma_{s I}(\underline{x}, E) P\left(E, \underline{\omega}, E^{\prime}, \underline{\omega}^{\prime} ; \underline{x}\right) \mathrm{d} E \mathrm{~d} \underline{\omega} ;
$$

and, for $x \in I I$,

$$
\Sigma_{s I I}\left(\underline{x}, E^{\prime}\right)=\iint \frac{\psi^{\infty}(\underline{x}, E, \underline{\omega})}{\psi^{\infty}\left(\underline{x}, E^{\prime}, \underline{\omega}^{\prime}\right)} \Sigma_{s I I}(\underline{x}, E) P\left(E, \underline{\omega}, E^{\prime}, \underline{\omega^{\prime}} ; \underline{x}\right) \mathrm{d} E \mathrm{~d} \underline{\omega} .
$$

We notice that these equations are the transport equations for the flux $\psi^{\infty}(\underline{x}, \mathrm{E}, \underline{\omega})$ in a source-free infinite medium of Region I and Region II scatterers, respectively. Since such a flux is isotropic and spatially constant, we may write

$$
\begin{aligned}
\psi^{\infty}(\underline{x}, E, \underline{\omega}) & =\frac{1}{4 \pi} \psi_{I}^{\infty}(E), \underline{x} \in I \\
& =\frac{1}{4 \pi} \psi_{I I}^{\infty}(E), \underline{x} \in I I .
\end{aligned}
$$


If we assume scattering is elastic and isotropic in the CM system, both in I and II, then writing Equation 4 in the $\mathrm{CM}$ system gives

$$
\begin{aligned}
P\left(E, \underline{\omega}, E^{\prime}, \underline{\omega}^{\prime} ; \underline{x}\right) & =\frac{1}{E\left(1-\alpha_{I}\right)} \delta\left[\mu_{c}-\frac{2 E^{\prime}}{E\left(1-\alpha_{I}\right)}+\frac{1+\alpha_{I}}{1-\alpha_{I}}\right], \underline{x} \in I \\
& =\frac{1}{E\left(1-\alpha_{I I}\right)} \delta\left[\mu_{c}-\frac{2 E^{\prime}}{E\left(1-\alpha_{I I}\right)}+\frac{1+\alpha_{I I}}{1-\alpha_{I I}}\right], \underline{x} \in I I .
\end{aligned}
$$

Then Equation 28 becomes

$$
\Sigma_{S I}\left(E^{\prime}\right) \psi_{I}^{\infty}\left(E^{\prime}\right)=\int_{E^{\prime}}^{E^{\prime} / \alpha_{I}} \frac{\Sigma_{s I}(E) \psi_{I}^{\infty}(E)}{E\left(1-\alpha_{I}\right)} \mathrm{dE}
$$

and Equation 29 becomes

$$
\Sigma_{\mathrm{sII}}\left(E^{\prime}\right) \psi_{\mathrm{II}}^{\infty}\left(E^{\prime}\right)=\int_{E^{\prime}}^{E^{\prime} / \alpha_{\mathrm{II}}} \frac{\Sigma_{\mathrm{sII}}(E) \psi_{\mathrm{II}}^{\infty}(\mathrm{E})}{E\left(1-\alpha_{\mathrm{II}}\right)} \mathrm{dE} .
$$

From this, it easily follows that

$$
\psi_{I}^{\infty}\left(E^{\prime}\right)=\frac{k}{\Sigma_{s I}\left(E^{\prime}\right) E^{\prime}}
$$

and

$$
\psi_{I I}^{\infty}\left(E^{\prime}\right)=\frac{k}{\sum_{s I I}\left(E^{\prime}\right) E^{\prime}}
$$

where $\mathrm{k}$ is any constant. If we choose $\mathrm{k}=1$ and substitute into Equation 26, we arrive at 


$$
\begin{aligned}
\underline{\omega} \cdot \underline{\nabla} \eta(\underline{x}, E, \underline{\omega})+\Sigma_{t}(\underline{x}, E) \eta(\underline{x}, E, \underline{\omega}) & =\iint \Sigma_{s}\left(\underline{x}, E^{\prime}\right) \frac{E^{\prime}}{E} P\left(E, \underline{\omega}, E^{\prime}, \underline{\omega^{\prime}} ; \underline{x}\right) \eta\left(\underline{x}, E^{\prime}, \underline{\omega}^{\prime}\right) d E^{\prime} d \underline{\omega}^{\prime} \\
& +\frac{Q^{*} *(\underline{x}, E,-\underline{\omega})}{\Sigma_{s}(\underline{x}, E) E},
\end{aligned}
$$

where

$$
\eta(\underline{x}, E, \underline{\omega})=\frac{F^{\dagger}(\underline{x}, E, \underline{\omega})}{\sum_{s}(\underline{x}, E) E} .
$$

Equation 35 for the transformed adjoint flux, $\eta$, is now in a form suitable for simulation by Monte Carlo without the use of weights at every collision. Indeed, precisely because $\frac{E^{\prime}}{E} P\left(E, \underline{\omega}, E^{\prime}, \underline{\omega}^{\prime} ; \underline{x}\right)$ is a properly normalized scattering kernel from energy $E^{\prime}$ to energy $E$, the integral term in Equation 35 is the scattering rate at $\left(\underline{x}, E^{\prime}, \underline{\omega}^{\prime}\right)$ in the flux $\eta$. While we have assumed that scattering is isotropic in the CM system, our conclusions remain valid as long as the scattering is elastic. This follows from the relationship $\partial \mu_{c} / \partial E_{U}=-\left(E_{L} / E_{U}\right) \partial \mu_{c} / \partial E_{L}$ which depends only on Equation 23. Notice that we have derived the physically intuitive altered kernel $\frac{E^{\prime}}{E} P$, based only on the requirement of unit weights at every collision. We conclude that no other transformation will accomplish this.

The only nonstandard feature of Equation 35 is that the flux, $\eta$, is discontinuous at the interface between Regions I and II, owing to the possible discontinuity in $\Sigma_{s}(\underline{x}, E)$ there. However,

$$
\Sigma_{\mathbf{s}}(\underline{\mathrm{x}}, \mathrm{E}) \eta(\underline{\mathrm{x}}, \mathrm{E}, \underline{\omega})=\frac{\mathrm{F}^{\dagger}(\underline{\mathrm{x}}, \mathrm{E}, \underline{\omega})}{\mathrm{E}}
$$

is continuous everywhere, so that the appropriate discontinuity in $\eta$ may be achieved by multiplying the weight of each particle by $\Sigma_{S I}(E) / \Sigma_{S I I}(E)$, when the particle passes from Region I into Region II, and by the inverse factor upon crossing from II into I.

The adjoint sampling procedures which result from this transformation may be described as follows: a starting direction, $\underline{\omega}_{\mathrm{o}}$, is chosen from an isotropic distribution, $\underline{x}_{0}$ is selected uniformly in $R$, and $E_{0}$ is chosen from 


$$
\frac{Q * *\left(\underline{x}_{0}, E,-\underline{\omega}_{o}\right)}{\Sigma_{s}\left(\underline{x}_{0}, E\right) E} / \int \frac{Q * *\left(\underline{x}_{o}, E,-\underline{\omega}_{o}\right)}{\Sigma_{s}\left(\underline{x}_{0}, E\right) E} d E=\frac{\Sigma_{a R}(E)}{\Sigma_{s R}(E) E} / \int_{0}^{E} \frac{\Sigma_{a R}(E)}{\Sigma_{s R}(E) E} d E .
$$

A starting weight factor,

$$
4 \pi V_{R} \int_{0}^{E} \frac{\Sigma_{\text {max }}(E)}{\Sigma_{s R}(E) E} d E
$$

is assigned each particle. First-collision points are selected from $\mathrm{T}\left(\underline{x}_{0}, \underline{x}_{1} ; E_{0}, \underline{\omega}_{0}\right)$, and the history terminates with probability $\Sigma_{a}\left(\underline{x}_{1}, E_{0}\right) / \Sigma_{t}\left(\underline{x}_{1}, E_{0}\right)$. With probability $\Sigma_{\mathrm{s}}\left(\underline{x}_{1}, E_{0}\right) / \Sigma_{t}\left(\underline{x}_{1}, E_{0}\right)$, a new energy, $E_{1}$, and direction, $\underline{\omega}_{1}$, are chosen from $\left(E_{0} / E_{1}\right) P\left(E_{1}, \underline{\omega}_{1}, E_{0}, \underline{\omega}_{0} ; \underline{x}_{1}\right)$. The only weight factors are the ones occurring upon passing from one region into another having a different scattering cross section. In this fashion, the transformed adjoint flux, $\eta$, is constructed. Then one uses the relationships

$$
\begin{aligned}
\iint_{\mathrm{Q} * * F .} & =\iint \Sigma_{\mathrm{aR}_{\mathrm{R}}}(\mathrm{E}) \mathrm{F}(\underline{\mathbf{x}}, \mathrm{E}, \underline{\omega}) \\
& =\iint_{\mathrm{Q} F}{ }^{\dagger} \\
& =\iint_{\mathrm{Q} \eta / \psi^{\infty}} \\
& =\iint_{\mathrm{Q} \eta \Sigma_{\mathrm{s}} \mathrm{E}}
\end{aligned}
$$

to convert $\eta$ tallies into estimates of the absorption rate. That is, estimators of $\eta$ must be multiplied by the factor $\Sigma_{s} E$ in order to yield estimators of the absorption rate in $R$. It is evident that the scheme described previously may be immediately generalized to any number of regions containing elastic scatterers.

It seems apparent that most of the deleterious effects of the adverse weight factors, $W_{c}$ (Equation 12) $\times W_{T}$ (Equation 14), and $W_{c}^{\prime}$ (Equation 21), have been eliminated by the preceding transformation.' Numerical evidence in support of 
this will be offered in the next section. However, one should expect such a reduction in variance through an interpretation involving dual importance sampling (see, e.g., References 8 and 11). That is, the function $\psi^{\infty}(\underline{x}, E, \underline{\omega})$ of Equation 30 , which accomplishes the transformation, may be viewed as a region-dependent dual-importance function, since the flux, F, is an appropriate importance function for solution of adjoint equations, and since $\psi^{\infty}$ is an approximation to $F$. Despite the discontinuity in $\psi^{\infty}$ (which, of course, is not present in F), the flux, $\psi^{\infty}$, will be of a good approximation to $F$ deep in the interior of any region. To this extent, one might anticipate variance reduction to be accomplished through the use of the transformation via $\eta$. This was the same philosophy Spanier and Gelbard successfully applied in solving multigroup thermal problems through solutions of transformed adjoint equations. 
TABLE 1

CONSTANT CROSS-SECTION RESULTS

\begin{tabular}{l|l|l|l|l|l}
\hline \hline \multicolumn{2}{c|}{ Direct } & \multicolumn{2}{|c|}{$\begin{array}{c}\text { Untransformed } \\
\text { Adjoint }\end{array}$} & \multicolumn{2}{|c}{$\begin{array}{c}\text { Transformed } \\
\text { Adjoint }\end{array}$} \\
\hline $1-\mathrm{p}$ & $\sigma_{\mathrm{R}}(\%)$ & \multicolumn{1}{|c|}{$1-\mathrm{p}$} & $\sigma_{\mathrm{R}}(\%)$ & \multicolumn{1}{c}{$1-\mathrm{p}$} & $\sigma_{\mathrm{R}}(\%)$ \\
\hline & & 0.004468 & 0.4118 & 0.004473 & 0.02910 \\
& & 0.017827 & 0.3946 & 0.01779 & 0.04511 \\
& & 0.06889 & 0.4208 & 0.06893 & 0.1045 \\
& & 0.1010 & 0.4204 & 0.10126 & 0.1343 \\
& & 0.1323 & 0.4424 & 0.1322 & 0.1441 \\
0.2955 & 0.697 & 0.2951 & 0.4024 & 0.2946 & 0.2315 \\
0.4031 & 0.590 & 0.3640 & 0.4488 & 0.4933 & 0.3495 \\
0.4978 & 0.453 & 0.4952 & 0.4958 & 0.5692 & 0.4284 \\
0.6336 & 0.334 & 0.6402 & 0.5501 & 0.6327 & 0.4742 \\
0.72904 & 0.278 & 0.73335 & 0.65256 & 0.73175 & 0.5580 \\
0.76468 & 0.240 & 0.76820 & 0.7321 & 0.76829 & 0.6635 \\
0.7988 & 0.203 & 0.80510 & 0.7423 & 0.8036 & 0.6537 \\
\hline
\end{tabular}

Figure 1.

Comparison of Relative Errors, Constant-Cross-Section Case

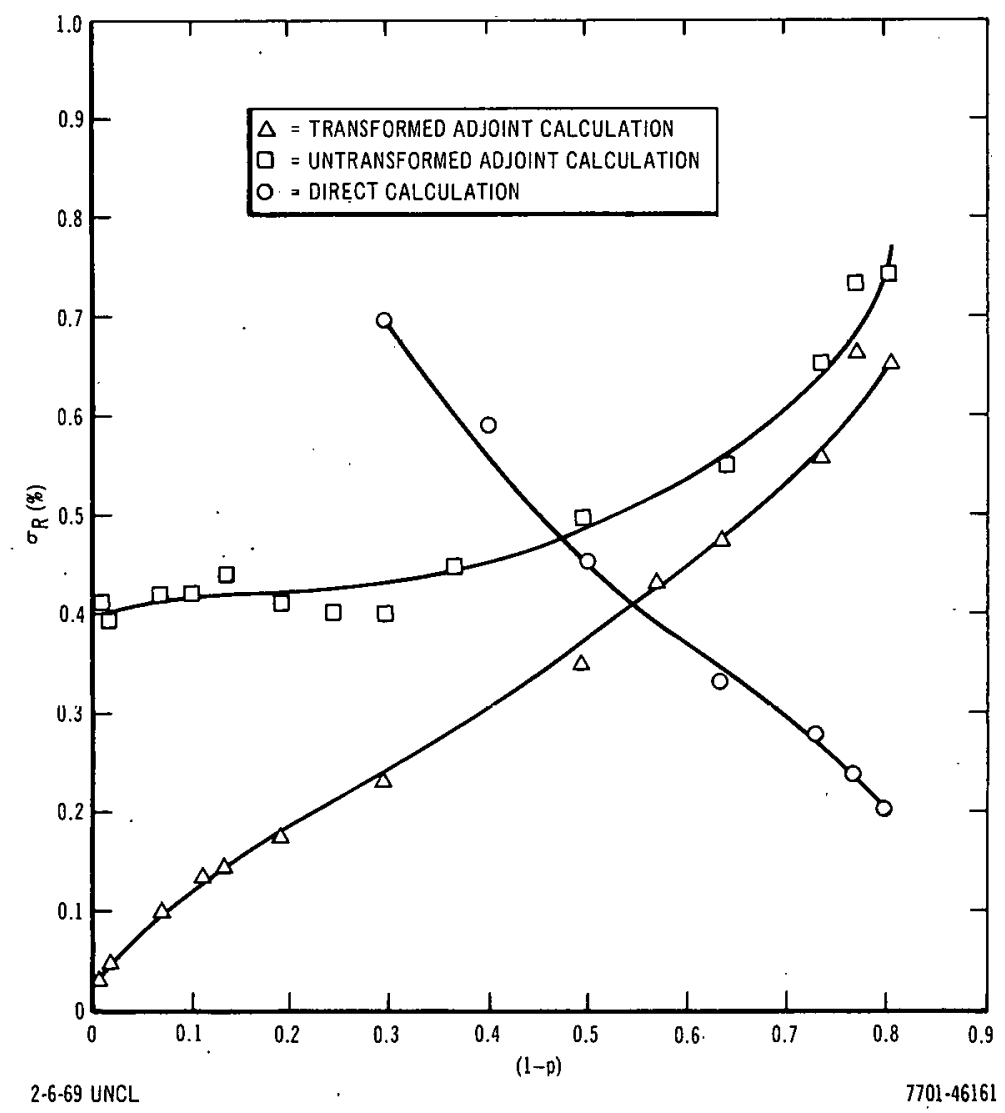

AI-AEC - 12774 


\section{NUMERICAL RESULTS}

A computer program, STRIFE, was written to compare the performance of the transformed adjoint method with that of an analog adjoint method, as well as to explore the range of problems in which an adjoint method is preferable to a direct method.

A constant-cross-section case was examined first, in order to easily compare direct, analog adjoint, and transformed adjoint methods. A two-region repetitive slab lattice, consisting of a graphite moderating region and a highly absorbing region containing a single isotope of large, but finite mass, was selected. By varying the thickness of the absorbing region, several problems with a range of "resonance" escape probabilities were studied. These results are shown in Figure 1 (based on Table 1), in which the relative standard deviation per history (in percent) is plotter against ( $1-\mathrm{p}$ ), the "resonance" absorption probability. As can be seen, when $(1-p)$ is very small, the advantages of adjoint over direct methods are apparent. Of even greater significance is the fact that the advantage of the transformation increases with decreasing $(1-p)$ to such an extent that accurate estimates of $(1-p)$ in this range may be obtained economically. The running times obtained reflect an additional advantage of adjoint over direct methods, namely, the shorter time per history, due primarily to the fact that adjoint particles are born with a distribution of energies, rather than at one end of the energy range. For the problems studied, this additional advantage was worth a factor of $\sim 1.7$ (not shown in Figure 1 or Table 1). The transformed adjoint method is 200 times as efficient as the analog adjoint method for small $(1-p)$, and still 3 times as efficient for $(1-p)=0.3$. Figure 2 shows the advantage (in time) resulting from the transformation for a wide range of $(1-p)$. Notice that, while the advantage diminishes considerably as ( $1-p)$ increases, so does the need to perform the calculation by using adjoint techniques.

Figure 3 and Table 2 show a comparison of direct and transformed adjoint results for a practical two-region problem with a graphite moderator and a gold resonance absorber. In such a problem, the energy dependence of the resonance absorber cross section gives rise to considerable latitude in the choice of sampling procedure for adjoint source energies. This energy dependence also tends 


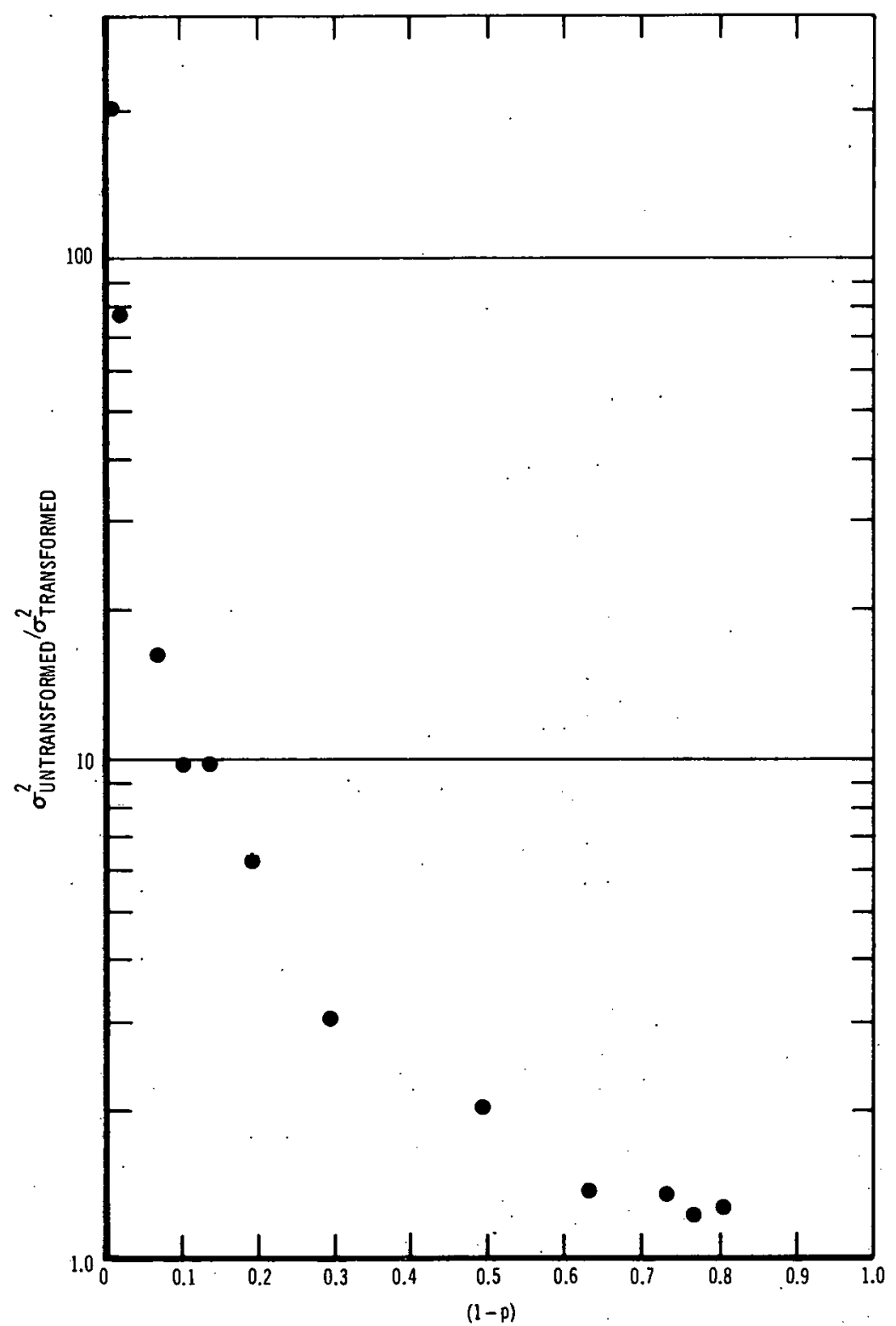

$2-669$ UNCL

77.01 .46162

Figure 2. Computational Advantage of Adjoint Transformation 
TABLE 2

ENERGY-DEPENDENT CROSS-SECTION RESU LTS

\begin{tabular}{l|r|l|l}
\hline \multicolumn{2}{c|}{ Direct } & \multicolumn{2}{c}{$\begin{array}{c}\text { Transformed } \\
\text { Adjoint }\end{array}$} \\
\hline $11-\mathrm{p}$ & $\sigma_{\mathrm{R}}(\%)$ & \multicolumn{1}{c}{$1-\mathrm{p}$} & $\sigma_{\mathrm{R}}(\%)$ \\
\hline 0.0056926 & 13.23 & 0.006543 & 1.79 \\
0.026082 & 5.62 & 0.02598 & 1.75 \\
0.046006 & 4.06 & 0.04899 & 1.86 \\
0.16254 & 2.29 & 0.1615 & 2.20 \\
0.24405 & 1.73 & 0.2453 & 2.12 \\
0.35103 & 1.28 & 0.32495 & 2.78 \\
\hline
\end{tabular}

Figure 3.

Comparison of Relative Errors, Two-Region Gold Resonance Problem

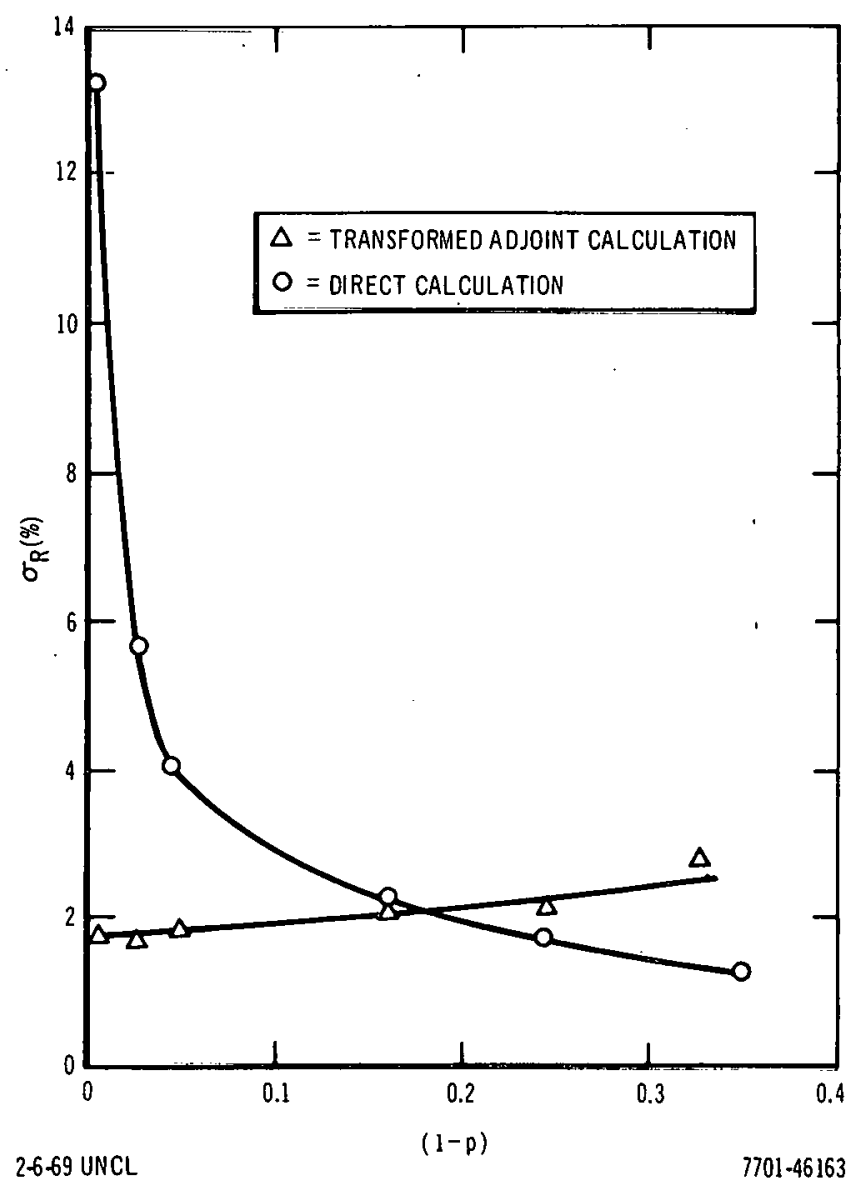


to reduce the relative efficiency of adjoint vs direct procedures. The results shown in Figure 3 and Table 2 were obtained by forcing half the adjoint particles to start inside an energy interval slightly larger than an interval containing the dominant gold resonance. Even with such a relatively simple procedure, the advantage of transformed adjoint to direct method for small ( 1 - p) is impressive. 


\section{SUMMARY AND CONCLUSIONS}

This report has described an adjoint technique which appears to be very useful for solving problems in the slowing-down region below the threshhold for inelastic scattering. The physically intuitive basis for this technique is by means of an exact reversal of direct Monte Carlo random walks, including the preservation of scattering angles. This idea was previously used in a combined direct-adjoint Monte Carlo calculation of resonance integrals. ${ }^{(13)}$ We have shown that this reversal may be also accomplished by a transformation of the adjoint transport equation by means of a discontinuous importance function which, in each region of the problem, is the flux in an infinite medium of scatterer appropriate for that region. From the latter point of view, this transformation may be seen to be a logical extension to continuous energies of the adjoint multigroup formulation of Spanier and Gelbard.

In addition to the variance reduction it achieves, the principal advantages of the adjoint technique described here stem from its ease of implementation; computational speed, and its ability to utilize the same nuclear data as an equivalent direct Monte Carlo calculation.

This new technique considerably expands the range of problems tractable by Monte Carlo. It seems particularly well suited, for example, to the analysis of foil experiments; and computational times appear to be sufficiently low to permit parametric studies to be performed economically. ${ }^{*}$

*Typical running times for transformed adjoint problems were 1 min or less on the CDC-6600. 


\section{REFERENCES}

1. R. R. Coveyou, V. R. Cain, and K. J. Yost; "Adjoint and Importance in Monte Carlo Application," Nucl. Sci. and Eng., 27 (1967) pp 219-234

2. L. Levitt, "The Use of Self-Optimized Exponential Biasing in Obtaining Monte Carlo Estimates of Transmission Probabilities," Nucl. Sci. and Eng. , 31 (1968) pp 500-504

3. J. Spanier, "An Analytic Approach to Variance Reduction," Submitted to SIAM J. Appl. Math.

4. V. R. Cain, "Application of $S_{N}$ Adjoint Flux Calculation to Monte Carlo Biasing," Trans. ANS, 10 (1967) p 399

5. M. Leimdörfer, "On the Transformation of the Transport Equation for Solving Deep Penetration Problems by the Monte Carlo Method," Trans. Chalmers Univ. of Tech., Gothenberg, Sweden, No. 286 (1964)

6. H. Kahn, "Applications of Monte Carlo," AECU-3259 (1954)

7. C. W. Maynard, "An Application of the Reciprocity Theorem to the Acceleration of Monte Carlo Calculations," Nucl. Sci. and Eng., 10 (1961) p 97

8. J. Spanier, "A Unified Approach to Monte Carlo Methods and an Application to a Multigroup Calculation of Absorption Rates," SIAM Review, $\underline{4}$ (1962) pp $115-134$

9. E. M. Gelbard, H. R. Ondis, and J. Spanier, "MARC - A Multigroup Monte Carlo Program for the Calculation of Capture Probabilities," WAPD-TM-273 (1962)

10. M. H. Kalos, "Monte Carlo Integration of the Adjoint Gamma-Ray Transport Equation," Nucl. Sci. and Eng., 33 (1968) pp 284-290

11. J. Spanier and E. M. Gelbard, Monte Carlo Principles and Neutron Transport Problems, Addison-Wesley Publishing Co., (London and Reading, Mass., 1969)

12. L. B. Levitt, "A New Use of Adjoints in the Monte Carlo Estimation of Resonance Integrals," NAA-SR-12494, (September 5, 1967)

13. G. Goertzel and M. H. Kalos, Progress in Nuclear Energy, D. J. Hughes, Ed., Series I, 2 (Pergamon Press, New York, 1958) pp 315-369 


\section{SAMPLING FROM THE COLLISION DENSITY EQUATION}

The integral Boltzmann equation for the neutron collision density is

$$
\begin{aligned}
\psi(E, \omega, x) & =\int \psi\left(E^{\prime}, \omega^{\prime}, x^{\prime}\right) C\left(E^{\prime}, \omega^{\prime}, E, \omega ; x^{\prime}\right) \mathrm{T}\left(x^{\prime}, x ; E, \omega\right) d E^{\prime} \mathrm{d} \omega^{\prime} \mathrm{d} x^{\prime} \\
& +\int Q\left(E, \omega, x^{\prime}\right) \mathrm{T}\left(x^{\prime}, x ; E, \omega\right) \mathrm{d} x^{\prime}
\end{aligned}
$$

If one wishes to calculate a reaction rate in a small region, $\mathrm{V}$, represented by the integral

$$
I=\int_{V} \psi(E, \omega, x) g(E, \omega, x) d E d x
$$

it can be shown that one can, instead, solve an equation adjoint to Equation A-1:

$$
\begin{aligned}
\psi *(E, \omega, x) & =\int \psi *\left(E^{\prime}, \omega^{\prime}, x^{\prime}\right) C\left(E, \omega, E^{\prime}, \omega^{\prime} ; x\right) T\left(x, x^{\prime} ; E^{\prime}, \omega^{\prime}\right) d E^{\prime} d \omega^{\prime} d x^{\prime} \\
& +g(E, \omega, x)
\end{aligned}
$$

in which the source term is the reaction probability per collision in $V$, and evaluate an integral

$$
I *=\iint \psi *(E, \omega, \mathbf{x}) Q\left(E, \omega, \mathbf{x}^{\prime}\right) \mathrm{T}\left(\mathbf{x}^{\prime}, \mathbf{x} ; E, \omega\right) \mathrm{d} \mathbf{x}^{\prime} \mathrm{d} E \mathrm{~d} \omega \mathrm{d} \mathbf{x},
$$

since it can be shown that

$$
I *=I \text {. }
$$

Direct mode sampling is accomplished by first selecting initial coordinates of a particle from the source, $S\left(E_{1}, x_{0}\right)$, and then moving the particle to its first collision point by selecting this point from $T\left(x_{0}, x_{1} ; E_{1}\right)$. The energy change then occurs by selecting $E_{2}$ from $C\left(E_{1}, E_{2} ; x_{1}\right)$. Sampling then alternates back 
to $T$, then $C$, etc., until such a time that the history terminates or enters the detector region. When this occurs, the product of the particle's statistical weight and the reaction probability, is tallied as an estimate of $I$.

Adjoint mode sampling starts in the detector region by sampling initial coordinates from the reaction probability, $g\left(E_{0}, \omega_{0}, x_{0}\right)$. One then proceeds by sampling from $T$, then $C$, etc., until a history terminates; or, if it enters the direct source region, an estimate of $I *$, which equals $I$, is tallied.

Comparison of Equation A-1 with Equation 1 reveals the following differences:

1) The transport kernel is $T\left(x^{\prime}, x ; E, \omega\right)$ instead of $T\left(x^{\prime}, x ; E^{\prime}, \omega^{\prime}\right)$

2) The collision kernel is $C\left(E^{\prime}, \omega^{\prime}, E, \omega ; x^{\prime}\right)$ instead of $C\left(E^{\prime}, \omega^{\prime}, E, \omega ; x\right)$

3) The source term is no longer simply $Q(x, E, \omega)$.

Similarly, comparing Equation A-3 with Equation 6 reveals corresponding differences in the transport and collision kernels, and leads to a simpler adjoint source term. Equation A-4 corresponds to Equation 7.

If we introduce

$$
C *\left(E, \omega, E^{\prime}, \omega^{\prime} ; x\right)=\frac{E^{\prime}}{E} P\left(E, \omega, E^{\prime}, \omega^{\prime} ; x\right)
$$

and

$$
T *\left(x, x^{\prime} ; E^{\prime}, \omega^{\prime}\right)=\frac{\Sigma_{t}\left(x, E^{\prime}\right)}{\Sigma_{t}\left(x^{\prime}, E^{\prime}\right)} T\left(x, x^{\prime} ; E^{\prime}, \omega^{\prime}\right)
$$

and let

$$
g(E, \omega, x)=\frac{\Sigma_{a}(E, x)}{\Sigma_{. t}(E, x)} X_{V}(E, x),
$$

where

$$
X_{V}=1 \text { if, and only if, }(x, E) \in V\left(X_{V}=0 \text {, otherwise }\right) \text {, }
$$

and then define

$$
\theta *(E, \omega, x)=\frac{\Sigma_{t}(E, x) \psi *(E, \omega, x)}{\Sigma(E, x) E},
$$


we may write

$$
\begin{aligned}
\theta *(E, \omega, \mathbf{x}) & =\int \theta *\left(E^{\prime}, \omega^{\prime}, x^{\prime}\right) \mathrm{W}_{\theta} \mathrm{C} *\left(\mathrm{E}, \omega, \mathrm{E}^{\prime}, \omega^{\prime} ; \mathbf{x}\right) \mathrm{T} *\left(\mathbf{x}, \mathrm{x}^{\prime} ; \mathrm{E}^{\prime}, \omega^{\prime}\right) \mathrm{d} \mathrm{E}^{\prime} \mathrm{d} \omega^{\prime} \mathrm{d} \mathbf{x}^{\prime} \\
& +\frac{\Sigma_{\mathrm{a}}(\mathrm{E}, \mathrm{x}) \mathrm{X}_{\mathrm{V}}(\mathrm{E}, \mathbf{x})}{\Sigma_{\mathrm{s}}(\mathrm{E}, \mathrm{x}) \mathrm{E}}
\end{aligned}
$$

where

$$
\begin{array}{r}
\mathrm{W}_{\theta}=\frac{\Sigma_{s}\left(E^{\prime}, \mathrm{x}^{\prime}\right)}{\Sigma_{t}\left(E^{\prime}, \mathrm{x}\right)} \text { or } \frac{\Sigma_{\mathrm{s}}\left(E^{\prime}, \mathrm{x}^{\prime}\right)}{\Sigma_{t}\left(E^{\prime}, \mathrm{x}^{\prime}\right)} \times \frac{\Sigma_{t}\left(E^{\prime}, \mathrm{x}^{\prime}\right)}{\Sigma_{t}\left(E^{\prime}, \mathrm{x}\right)} \\
\cdot \quad \text { or } \frac{\Sigma_{s}\left(E^{\prime}, \mathrm{x}\right)}{\sum_{t}\left(E^{\prime}, \mathrm{x}\right)} \times \frac{\Sigma_{s}\left(E^{\prime}, \mathrm{x}^{\prime}\right)}{\Sigma_{s}\left(E^{\prime}, \mathrm{x}\right)} .
\end{array}
$$

This weight is then either the scattering probability at $\left(E^{\prime}, x^{\prime}\right)$, where it would occur in the corresponding direct history, times a ratio of total cross sections, which is unity except at boundary crossings; or it is the scattering probability at $\left(E^{\prime}, x\right)$, the point where the adjoint particle enters collision, times the ratio of scattering cross sections, which departs from unity only at boundary crossings.

Looking at the test problem in detail, as shown in the following diagram,

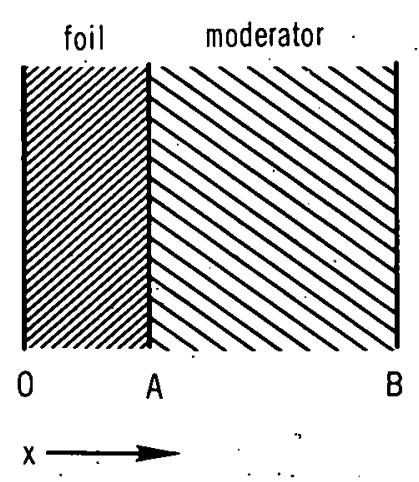

we wish to find the absorption rate $(1-p)$ in the region of phase space

$$
0<\mathrm{x}<\mathrm{A}, \quad \mathrm{E}_{\min }<\mathrm{E}<\mathrm{E}_{\max }
$$




$$
Q\left(E, x^{\prime}\right)=0 \text { if } 0<x^{\prime}<A
$$

and

$$
\alpha_{\operatorname{m}} E_{\max }<\dot{E}<E_{\max }
$$

where

$$
\alpha_{m}=\left(\frac{\mathfrak{a}_{m}-1}{\mathfrak{A}_{m}+1}\right)^{2}
$$

and

$$
\mathbb{Q}_{\mathrm{m}}=\text { mass number of the material in the moderator }\left(\mathrm{x}^{\prime}>\mathrm{A}\right) \text {. }
$$

$$
Q\left(E, \omega, x^{\prime}\right) \propto \int_{E_{\max }}^{E / \alpha} \frac{1}{E^{\prime}} \frac{d E^{\prime}}{E^{\prime}\left(1-\alpha_{m}\right)} ; \int Q\left(E, \omega, x^{\prime}\right) d E d \omega d x^{\prime}=1
$$

from which

$$
\begin{aligned}
& Q\left(E, \omega, x^{\prime}\right)=\frac{\left(E-\alpha_{m} E_{m a x}\right)}{\xi E E_{\text {max }}\left(1-\alpha_{m}\right)} \cdot \frac{1}{(B-A)}, \\
& k \frac{B-A}{1-\alpha_{m}} \int_{\alpha_{m} E_{\text {max }}}^{E_{\max }}\left(\frac{1}{E_{\max }}-\frac{\alpha_{m}}{E}\right) d E=1, \\
& k=\frac{1-\alpha_{m}}{B-A} \cdot \frac{1}{\left(1-\alpha_{m}\right)-\alpha_{m} \ln \frac{1}{\alpha_{m}}} \equiv \frac{1}{\xi(B-A)} ;
\end{aligned}
$$

thus

$$
Q\left(E, \omega, x^{\prime}\right)=\frac{\left(E-\alpha_{m} E_{\text {max }}\right)}{\xi E E_{\text {max }}\left(1-\alpha_{m}\right)} \cdot \frac{1}{(B-A)}
$$

for

$$
\begin{gathered}
A<\mathrm{x}^{\prime}<\mathrm{B}, \\
\alpha \mathrm{E}_{\max }<\mathrm{E}<\mathrm{E}_{\max }
\end{gathered}
$$


Direct mode tallying, when simulating the integral transport equation for the collision density, estimates the integral shown in Equation A-2. In a direct mode Monte Carlo, one tallies $W(E, x) \frac{\Sigma_{a}(E, x)}{\Sigma_{t}(E, x)}$ at every collision in appropriate energy and spatial regions, or one can tally $W(E, x)$ with probability $\Sigma_{a}(E, x)$ $\frac{a}{\sum_{t}(E, x)}$

Tallying based on Equation A-4 is more complicated, and will now be examined in detail. Rewriting Equation A-4 as

where

$$
I *=\int \psi *(E, \omega, \mathbf{x}) S(E, \omega, \mathbf{x}) \mathrm{d} E \mathrm{~d} \omega \mathrm{d} \mathbf{x},
$$

$$
S(E, \omega, x)=\int Q\left(E, \omega, x^{\prime}\right) \mathrm{T}\left(x^{\prime}, \mathbf{x} ; E, \omega\right) \mathrm{d} \mathbf{x}^{\prime},
$$

one can see that, in our test problem;

$$
S(E, \omega, x)=\frac{1}{(B-A)} \cdot \frac{\left(E-\alpha_{m} E_{\text {max }}\right)^{\prime}}{\xi E E_{\text {max }}\left(1-\alpha_{m}\right)} \int_{A}^{B} T\left(x^{\prime}, x ; E, \omega\right) d x^{\prime}
$$

We now define

$$
\begin{aligned}
J(x, E) & =\int_{A}^{B} T\left(x^{\prime}, x ; E, \omega\right) d x^{\prime} \\
& =\frac{\Sigma_{t}(E, x)}{\Sigma_{t}\left(E, x^{\prime}\right)} \int_{A}^{B} T *\left(x, x^{\prime} ; E, \omega\right) d x^{\prime} .
\end{aligned}
$$

Then, if $x<A$,

$$
J(x, E)=\frac{P_{1} P_{2}}{P_{c}} \cdot \frac{\Sigma_{t}(x, E)}{\Sigma_{t}\left(x^{\prime}, E\right)}
$$

if $A<\mathbf{x}<B$,

$$
J(x, E)=1-\frac{P_{3} P_{4}}{P_{c}}
$$


where $\quad P_{1}=$ probability of reaching the moderator,

$$
\begin{aligned}
& P_{2}=\text { probability of colliding in the moderator, } \\
& P_{3}=\text { probability of reaching the absorbing region, } \\
& P_{4}=\text { probability of colliding in the absorbing region, } \\
& P_{c}=\text { probability of colliding anywhere in the cell. }
\end{aligned}
$$

Thus, at the $i^{\text {th }}$ collision,

$$
S\left(E_{i}, \omega_{i}, x_{i}\right)=\frac{1}{B-A} \cdot \frac{E_{i}-\alpha_{m} E_{\max }}{\xi E_{i} E_{\max }\left(I-\alpha_{m}\right)} \cdot J\left(E_{i}, x_{i}\right) .
$$

One could tally, at every $\left(E_{i}, x_{i}\right)$ for which $S\left(E_{i}, \omega_{i}, x_{i}\right) \neq 0$,

$$
W\left(E_{i}, x_{i}\right) F\left(E_{i}, x_{i}\right) S\left(E_{i}, \omega_{i}, x_{i}\right),
$$

where $W\left(E_{i}, x_{i}\right) F\left(E_{i}, x_{i}\right)$ is an estimate of $\psi *\left(E_{i}, \omega_{i}, x_{i}\right)$. But

$$
W^{\theta}\left(E_{i}, x_{i}\right) F^{\theta}\left(E_{i}, x_{i}\right)=W^{\theta}\left(E_{i+1}, x_{i+1}\right) \frac{\Sigma_{t}\left(E_{i}, x_{i+1}\right)}{\Sigma_{t}\left(E_{i}, x_{i}\right)} E_{i}
$$

and

$$
S\left(E_{i}, \omega_{i}, x_{i}\right)=Q\left(E_{i}, \omega_{i}, x_{i+1}\right) J\left(E_{i}, x_{i}\right)
$$

sampling from $J\left(E_{i}, x_{i}\right)$ can be accomplished by selecting $x_{i+1}$ and tallying only if $x_{i+1}>A$, weighting by $\Sigma_{t}\left(x_{i}, E_{i}\right) / \Sigma_{t}\left(x_{i+1}, E_{i}\right)$. Therefore, one can tally, at every $E_{i+1}$ for which $\alpha_{m} E_{\max }<E_{i}<E_{\max }$ and $A<x_{i+1}<B$, the quantity

$$
W^{\theta}\left(E_{i+1}, x_{i+1}\right) E_{i} S\left(E_{i}, \omega_{i}, x_{i+1}\right) .
$$

One can defer the tallying still further as follows: tally only at $\left(E_{i+1}, x_{i+1}\right)$ if $E_{i+1}>E_{\max }$ and $A<x_{i+1}<B$. To see that this is valid, let $P_{E_{\max }}$ be the probability of selecting an $E_{i+1}>E_{\max }$. 
Then

$$
P_{E_{\max }}=\int_{E_{\max }}^{E_{i} / \alpha} \frac{E_{i} d E_{i+1}}{E_{i+1}^{2}(1-\alpha)}
$$

Therefore,

$$
P_{E_{\max }}=E_{i} \frac{E_{i}-\alpha E_{\max }}{E_{i} E_{\max }(1-\alpha)}
$$

but this is

$$
\left.E_{i} Q\left(E_{i}, \omega_{i}, x_{i+1}\right) \xi(B-A) \quad \text { (see Equation } A-6\right) \text {. }
$$

Therefore, at such an $\left(E_{i+1}, x_{i+1}\right)$, tally

$$
\frac{w^{\theta}\left(E_{i+1}, x_{i+1}\right)}{\xi(B-A)}
$$

This is analogous to terminal tallying (in which a particle is allowed to be absorbed in the detector region) in a direct Monte Carlo simulation, in that the existing weight of an adjoint particle is tallied upon termination of a history in the direct source region.

It is this method of tallying which can lead to zero variance in the estimate when the actual flux in the moderator behaves exactly as the importance function implied by this method (see Equations 33 and 34 ). 


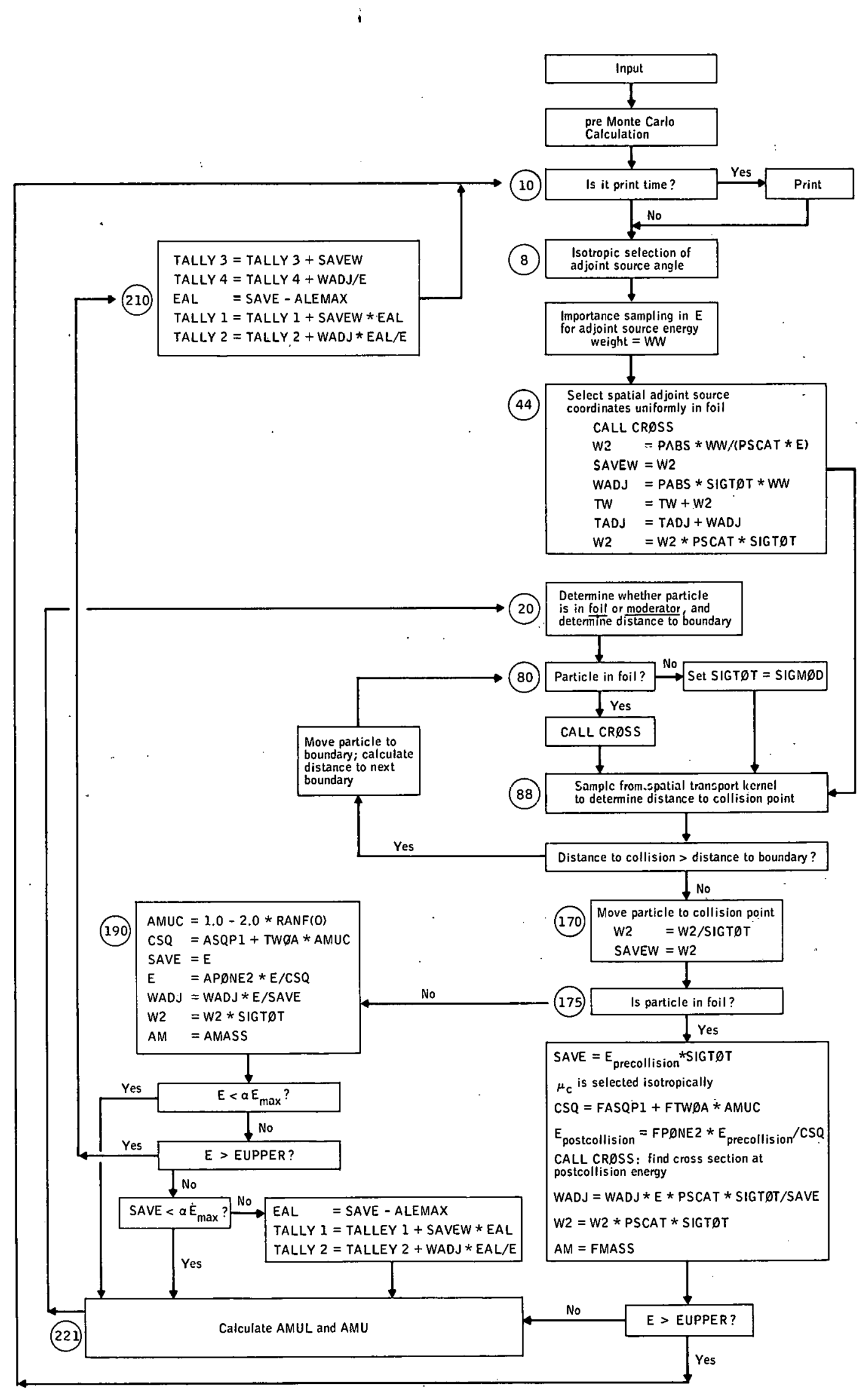

AI-AEC- 12774 


\section{APPENDIX II \\ STRIFE CODE}

The STRIFE code calculates the resonance escape probability (p) for a tworegion repetitive slab lattice consisting of a moderating region of thickness $B$, constant scattering cross section SIGMOD (zero absorption), mass AMASS, and a highly absorbing region of thickness A containing a single isotope of large mass FMASS. The STRIFE code uses the simulation procedures described in Appendix I.

Versions of the STRIFE code exist for the direct simulation method, the analog method, and the transformed adjoint method.

Since the code could be easily adapted, with minor modification, to the solution of practical problems involving reaction rates in localized regions, a flow chart and listing of the transformed adjoint version of the STRIFE code are provided. 
PROGRAM MAIN (INPUT, OUTPUT, TAPES=INPUT, TAPE6=OUTPUT)

COMMON RDUMTRNT XMU

COMMON EP(100),STRESN $(100)$, SERESN $(100)$

COMMON A,B,E,WOX,SB,ST,T2,XITAMU,CSQ,AMUE,AMUL,NBOT,NTEM,NTOP, TWO

1 A, RATIO, PSCAT, NHIST, DENOM, ALPHA, AMASS, ASQPI, TALLYS, TALLY1, TALLYZ,

2SIGMOD,SIGTOT,NTHIST, NBATCH,IESAVE, EUPPER, ERATTO,ELOWER, APONEZ,

3 ALEMAX

COMMON CUMXTTOOT

DIMENSION STA $(200)$,STB $(200)$, STAI $(200)$, STBI $(200)$

RNERANF TIT

IEND $=7.3$

READ $\left(5,500 T^{-N}\right.$ NTHIST,NBATCA,NTOP,NBOT

READ $(5,5002)$ A, B, FMASS, AMASS, DENF, DENM

READ $(5,5002)^{-1}$ STGMOO-

READ $(5,5002) \quad(E P(I), I=1$, IENO)

READ $(5,5002)$ (STRESN(T), $1=1$, IENO)

$\operatorname{READ}(5,5002)$ (SERESN (I), I 1 , IEND)

500 T FORMATTICT6r

5002 FORMAT.(6E 12.8$)$

48T FORMATTIH ,T4, 4 E20.81

$I B E G=4$

IENOM $=72$

$X H I=S T R E S N(I B E G)-S E R E S N(I B E G)$

DO'-480 TETBEG, TENDM

$X \operatorname{O}=\operatorname{STRESN}(I+1)-\operatorname{SERESN}(I+1)$

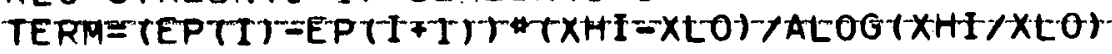

IF (I.EQ.IBEG) GO TO 450

CUMXTIT=CUMXTI-ITTERM

GO TO 400

$450^{\circ}$ CUMXTIBEGT $=$ TERM

$400 \times H I=X L O$

480 WRTTE $\left(6,481 \mathrm{~T}^{-}\right.$I,EP(I),COMX(I),-STRESNTI),-SERESNTI)

DO 3 I = I, IEND

SERESNII) = SERESN(I)/STRESNII

$3 \operatorname{STRESN}(1)=\operatorname{STRESN}(I) *$ DENF

SIGMOD-

IEND02 $=I E N D / 2$

DO 4 T $=1$ TENDO2

$K=I E N D+1-I$

DUM $=E P(I)$

$E P(I)=E P(K)$

EP(KT)=-DUM

DUM = STRESN (I)

STRESNTIT- $=-$ STRESNAKT

STRESN $(K)=$ DUM

DUM = SERESNII

$\operatorname{SERESN}(\mathrm{I})=\operatorname{SERESN}(K)$

4-SERESH(K)- =OUM

AI-AEC-12774 
$3333 T 2=B-A$

$\forall O t=A>T 2$

PSCAT $=.8$

STOF $=1000 . \triangle 0 E N F$

$P A B S=1.0-P S C A T$

STGTZ $=-72 * 51 G M O \theta$

$A S Q P 1=$ AMASS $* 2+1.0$

FASQP1- $=$ FMASS*2-7-1:0

TWOA $=2.0 *$ AMASS

FTWOA $=2.0$ FMASS

APONE2 $=($ AMASS + 1.0$) * 2$

FPONEZ =-TFMASS-1-50)*Z

EUPPER $=E P(N T O P)$

ELOWER- $=$ EP-(NOOT)

WRITE $(6,5101)$ NTHIST, NBATCH,NTOP, EUPPER, NBOT, ELOWER, A, B, FMASS,

I ARASSTOENF, OENM,STGMOO

5101 FORMAT IIHO, *TOT NO: HIST REQUESTED=*,I7,*, BATCH SIZE=*, I5,

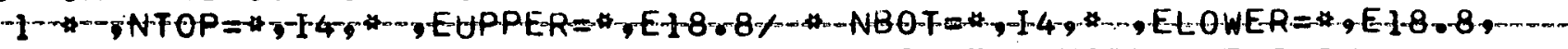

2 , $A(T H I C K N E S S$ FUEL $)=\star, E_{18.8, * B(T H I C K N E S S ~ M O D)}=\sharp, E 18.8 /$

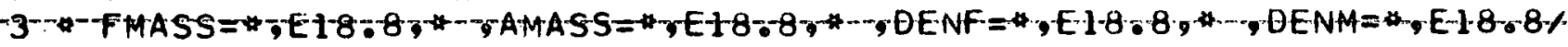

4 SIGMOD $=*, E 18.81$

ERATIO = EUPPER/ELOWER

DELU $=A L O G(E R A T I O)$

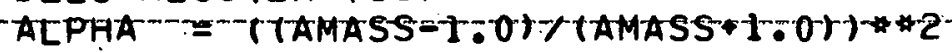

ALEMAX = EUPPER \#ALPHA

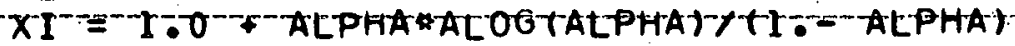

DENOM $=(1.0-A L P H A) * X I * E U P P E R$

WRTTE 18,9911 DELU,XI,OENOM

991 FORMAT $(1 H O, * D E L U=*, E 20.8, * X I=*, E 20.8, * D E N O M=*, E 20.8)$

NTEMM--- $=-0$

$A P D=A+1.0 E-10$

$A M D^{-}=-A=-1-O E=10$

$T 2 M D=T 2-1.0 E-10$

TALLYI $=0.0$

TALLY2 $=0.0$

TALTY $3=0.0$

TALLY $Y=0.0$

TAOJ $=0.0$

$T W=0.0$

$N+T S T=0$

NBB $=0$

NTOPPIONTOPFI

10 IF (NTEM.GE.NBATCH) GO TO 3000

NTEM =NTEM-I

NHIST $=$ NHIST +1

IESAVE $=$ NBOT+1

8 AMU $=1 \cdot-2 \cdot$ RANF $(0)$

IF (AMU.EO.0.01-'GO'TO'-8

$E=E L O W E R * E R A T I O *$ RANF $(0)$ 
$X=A$ RANF TOT

SIGTOT =S I GF

WZ $=P A B S T P S C A T$

SAVEW $=W 2$

WAOJIPABS*SIGTOT*E

$T W=T W+W 2$.

$T-A D J=T A B J+W A B J$

$W 2=W 2 * P S C A T * S I G T O T$

IF TAMU.GT.0.0, GO-TO- 14

$S B=-x$

GO-TO- 88

$14 S B=A-X$

GO-TO- 88

20 IF (X.GT.A) GO TO 60

22 IF (AMU.GT.0.0) GO-TO-40

$S B=-X$

$60-10-80$

$40 \mathrm{SB}=A-X$

GO $10-80$

60 IF (AMU.LT.0.0) GO TO 40

$S B=B-X$

80 SIGTOT $=$ SIGMOD

$\operatorname{IF}\left(X . G T \cdot A^{-}\right)^{-G O} \mathrm{TO}^{\circ} 88$

SIGTOT $=S I G F$

$88 \mathrm{ST}^{-}=-A[O G T R A N F$ COTITSIGTOT AMO

IF (AMU.LT.0.0) GO TO 180

IF (ST.GT.SB) GO TO 200

$170 \quad x=x+S T$

WE= TErSIOTOT

SAVEW=W2

T75 TF (X.GT-A)-GO FO 190

SAVE $=E * S I G T O T$

AMUC $=1.0-2.0$ RAINF (O)

CSO $F A S Q P 1+F T W O A * A M U C$

E=FPONEZ ETCSO

SIGTOT $=$ SIGF

WAOJ=WAOJ*E*PSCAT*STGFOT/SAVE

$W 2=W 2 \# P S C A T * S I G T O T$

$A M=F$ MASS

IF (E.GT.EUPPER) GO TO 10

GO-TO-221

180 IF (ST.LT.SB) GO TO 201

60-TO-170

190 AMUC $=1.0-2.0 \otimes R A N F(0)$

CSQ = ASOPI TWOA AHEC

$S A V E=E$

E- $=$ APONE-Z EE CSO

$W A D J=W A D J * E / S A V E$

$\forall 2=W 2 * S T G T O T$ 


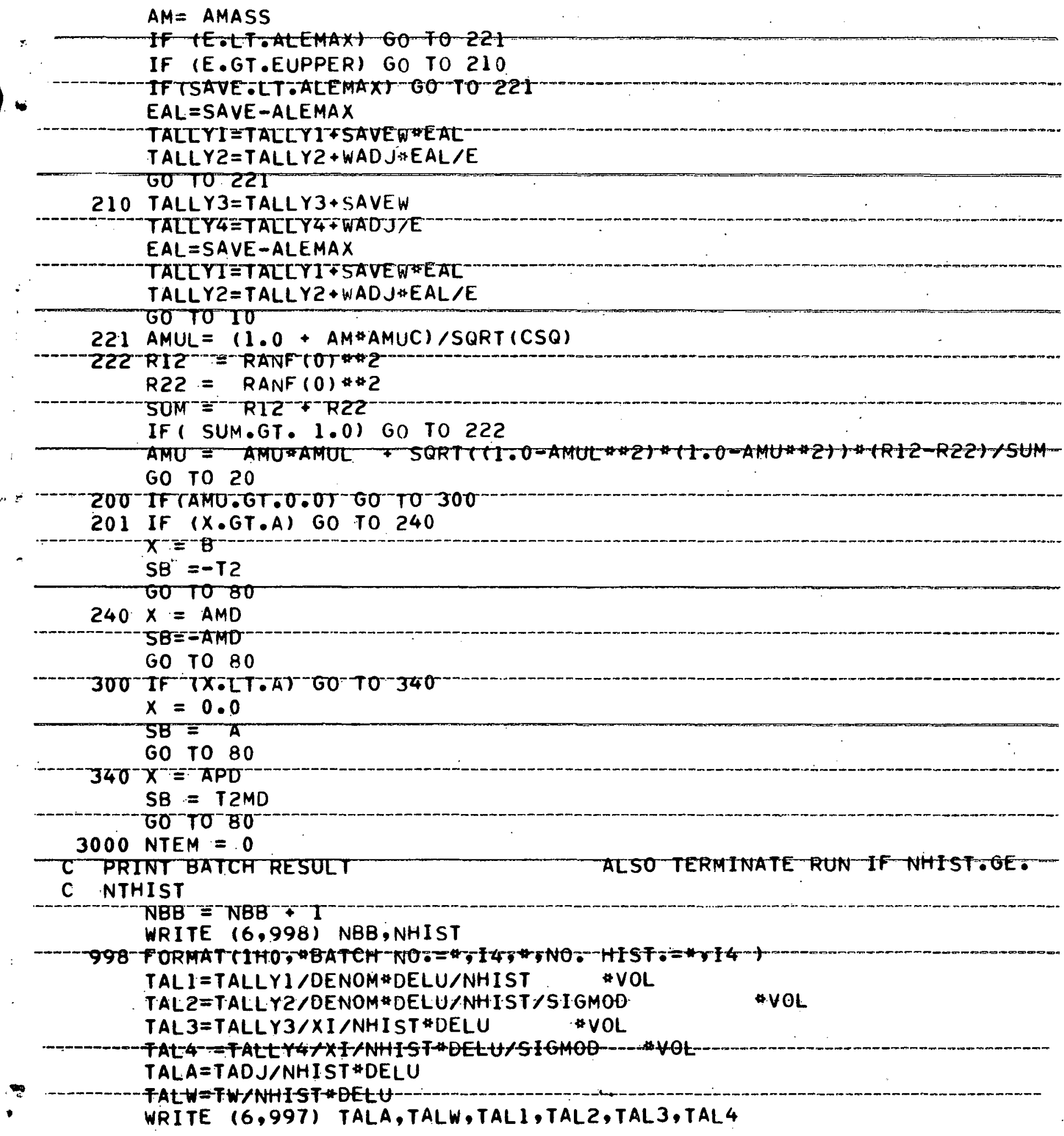




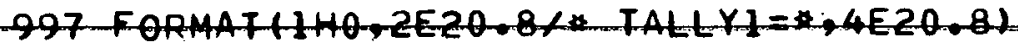

$S T A(N B B)=T A L 1$

STE TNBB-r $=$ TALT-3

IF (NHIST •LT.NTHIST) GO TO 10

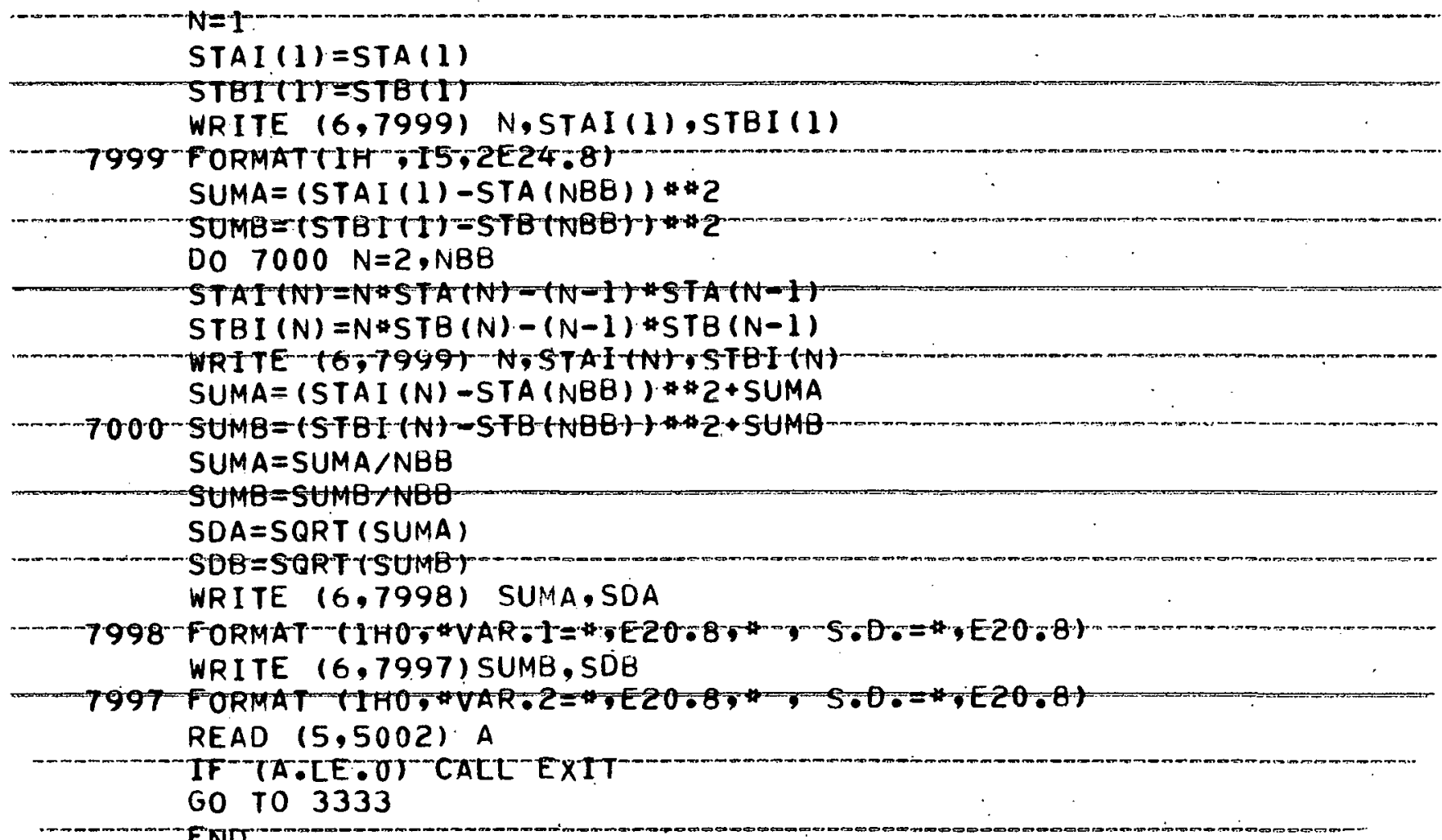
END 
(11) 